\title{
Johann Philipp von Wessenberg-Ampringen, admirateur réaliste à l'époque de Napoléon et de sa gloire
}

\section{Peter Heinrich von Wessenberg}

Traducteur : Christophe Didier

\section{OpenEdition}

Journals

Édition électronique

URL : http://journals.openedition.org/rbnu/1397

DOI : $10.4000 /$ rbnu. 1397

ISSN : 2679-6104

\section{Éditeur}

Bibliothèque nationale et universitaire de Strasbourg

\section{Édition imprimée}

Date de publication : 1 novembre 2015

Pagination : 72-95

ISBN : 9782859230616

ISSN : 2109-2761

\section{Référence électronique}

Peter Heinrich von Wessenberg, « Johann Philipp von Wessenberg-Ampringen, admirateur réaliste à l'époque de Napoléon et de sa gloire », La Revue de la BNU [En ligne], 12 | 2015, mis en ligne le 01 mars 2020, consulté le 13 décembre 2020. URL : http://journals.openedition.org/rbnu/1397 ; DOI : https:// doi.org/10.4000/rbnu.1397

\section{(c) (i) (9)}

La Revue de la BNU est mise à disposition selon les termes de la Licence Creative Commons Attribution - Pas d'Utilisation Commerciale - Partage dans les Mêmes Conditions 4.0 International. 


\section{- Ie derpotisme do Supoteon.}

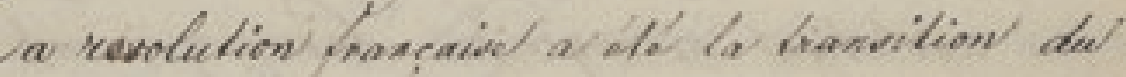

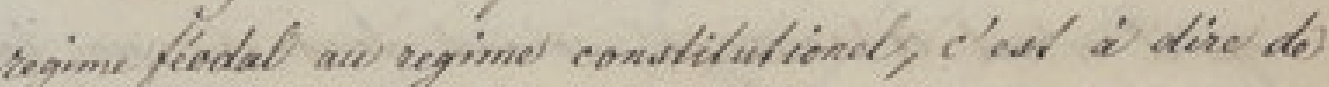

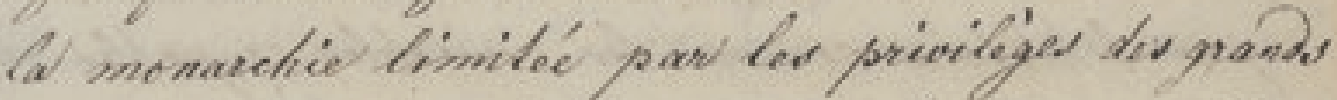

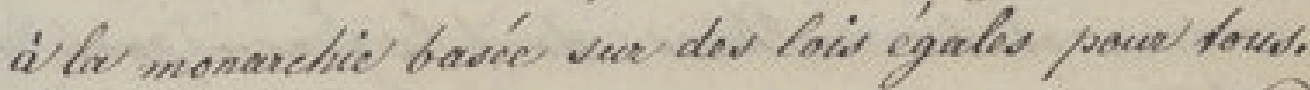

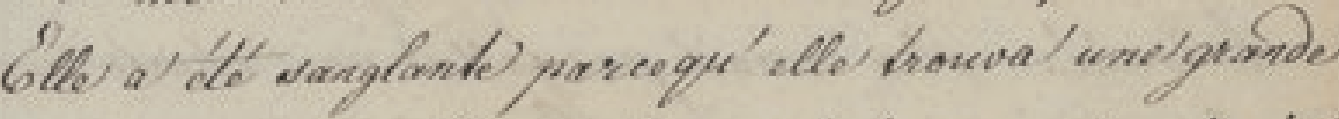

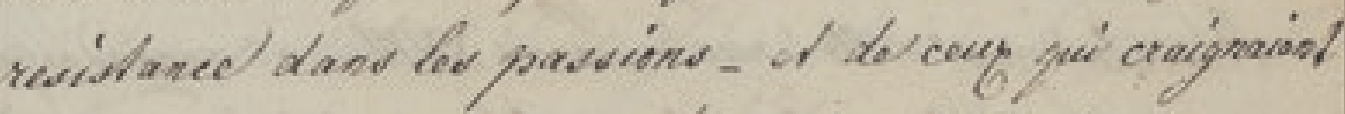

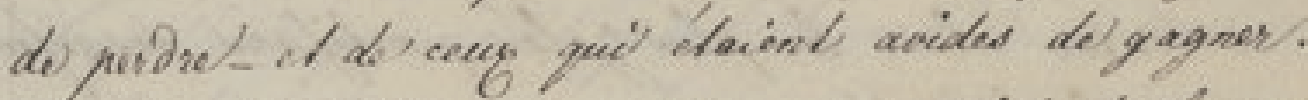

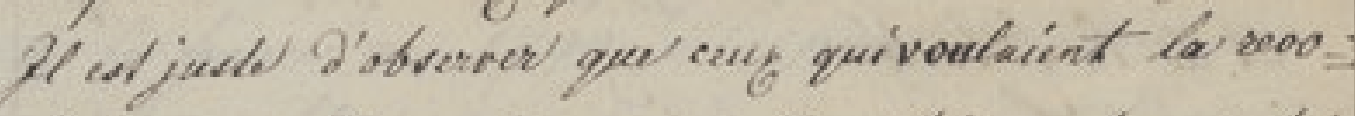

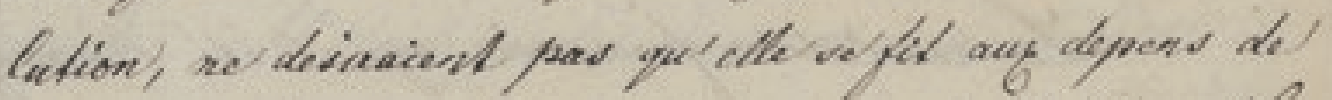

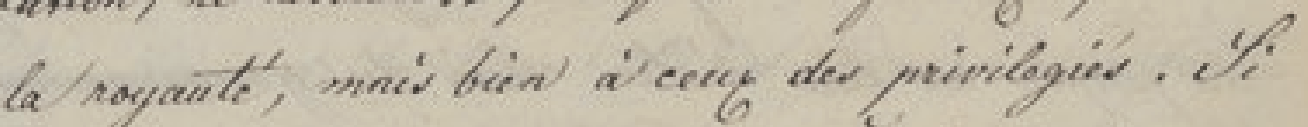

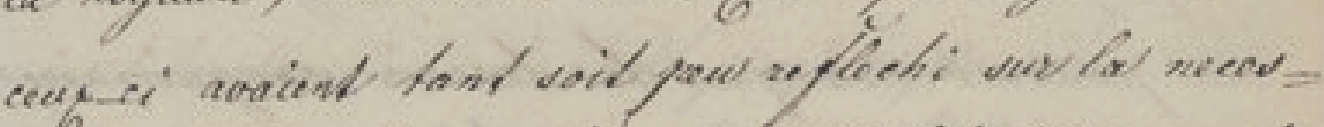

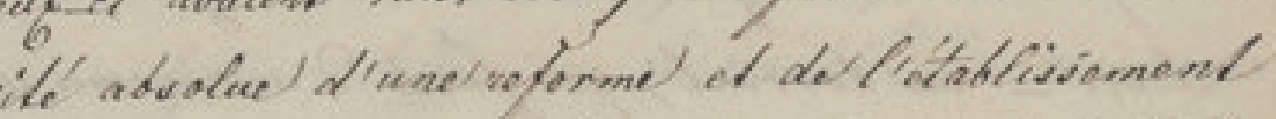

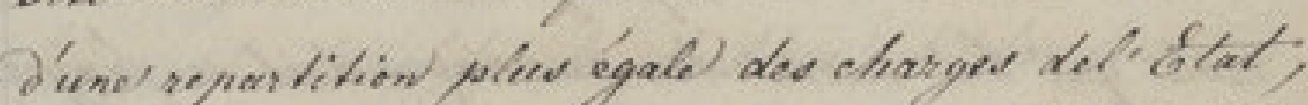

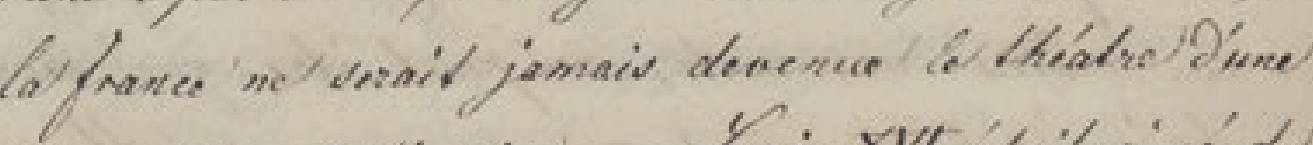

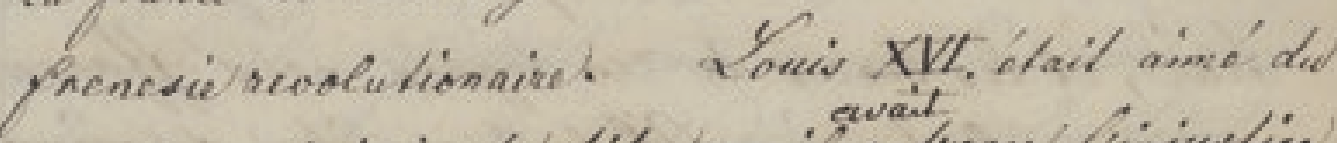

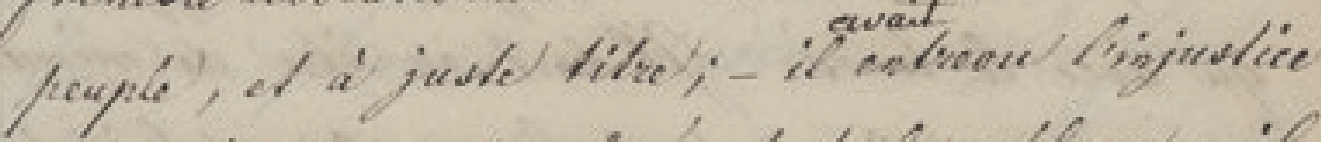

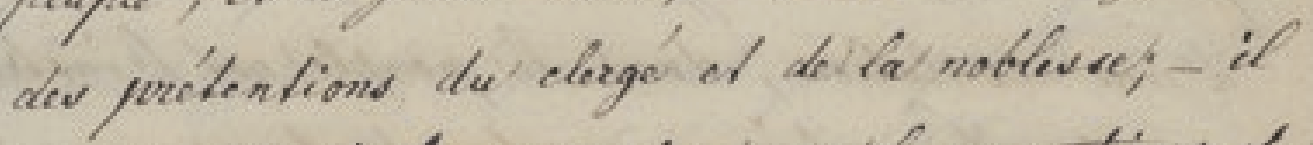

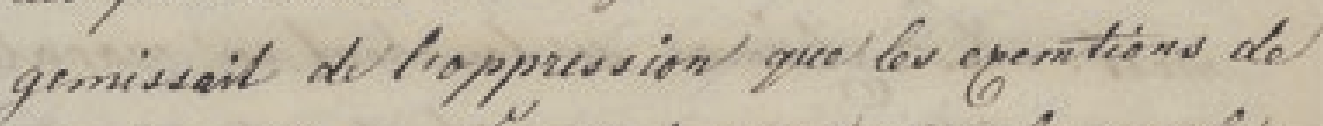

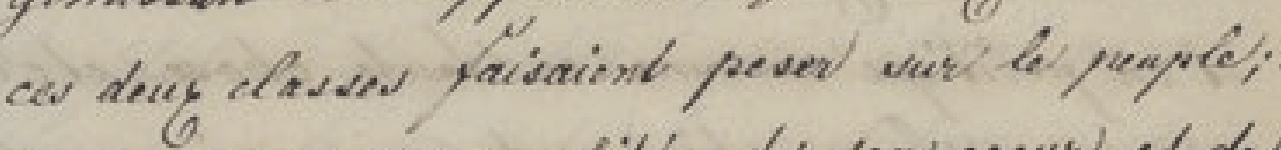

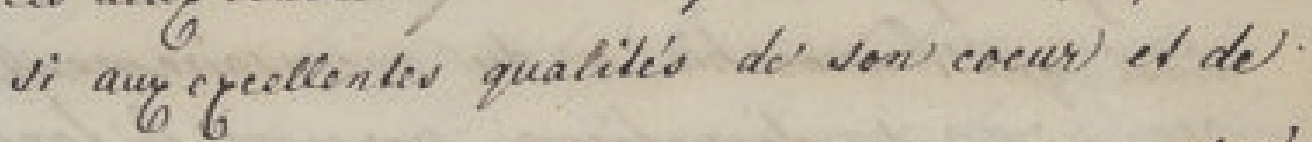




\section{JOHANN PHILIPP VON WESSENBERG-AMPRINGEN, ADMIRATEUR RÉALISTE À L'ÉPOQUE DE NAPOLÉON ET DE SA GLOIRE}

$\grave{\mathrm{A}}$ travers les lettres à son frère Ignaz Heinrich, le dernier administrateur de l'évêché de Constance, nous découvrons de nombreuses prises de position personnelles de Johann Philipp sur Napoléon ${ }^{\text {er }}$, sa vie, sa mort, sa personnalité, son rapport à la politique, son despotisme, mais aussi sur les causes de la Révolution, sur le roi Bourbon Louis XVI et sur la situation de la France en Europe. Les remarques qui suivent concernent un choix de textes issus des Fragmens politiques écrits par Johann Philipp (la plupart en français, quelques-uns en allemand), qui n'ont jamais été publiés et dont la BNU conserve le manuscrit original.

\section{Sur Le despotisme de Napoléon (1818)}

Johann Philipp était un des diplomates les plus lettrés de son temps. Il écrit ainsi à son frère Ignaz Heinrich en 1858, après la lecture des Mémoires d'André-François Miot, comte de Mélito : "Ce que Miot dit de Napoléon I $^{\text {er }}$ est caractéristique. Sa soif du pouvoir, sa dureté et son absence de scrupules étaient manifestes dès 1796 ".

Il faut savoir que Johann Philipp eut pendant presque douze ans des occasions de rencontrer personnellement Bonaparte et que ces rencontres eurent lieu aussi bien dans des circonstances exceptionnelles ou de situations peu banales, que dans des lieux d'une importance particulière, depuis la première grande audience du Premier Consul lors de sa médiation pour la Suisse en février 1803 à Saint-Cloud, jusqu'au quartier général de Napoléon à Saint-Dizier en 1814.
Le regard de Johann Philipp sur Napoléon est moins ambivalent, il est plus objectif dans ses jugements ou ses commentaires. Il confronte toujours la personne aux faits ; ainsi il note que l'intelligence du " consul " est digne d'admiration et de la même façon que sa vanité accroît son arbitraire. De fait, les mots de Wessenberg comportent toujours des réserves, comme par exemple lorsqu'il constate : " Je tremble encore. Mon espérance n'est toujours pas plus forte que ma crainte. L'empereur Bonaparte saura-t-il mieux assumer sa bonne fortune que le consul Napoléon ?" 
14.

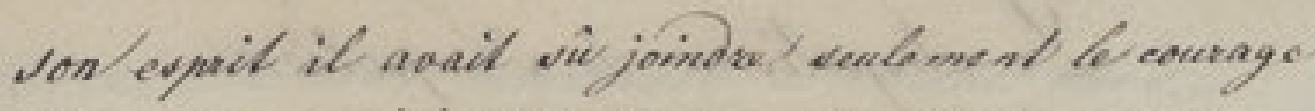

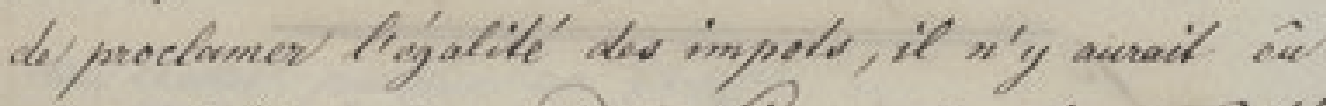

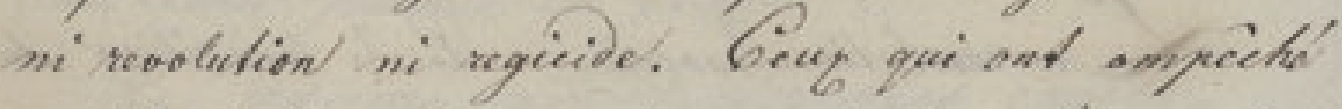

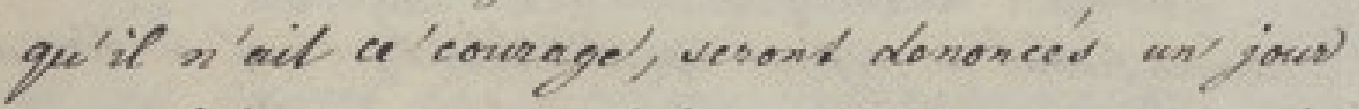

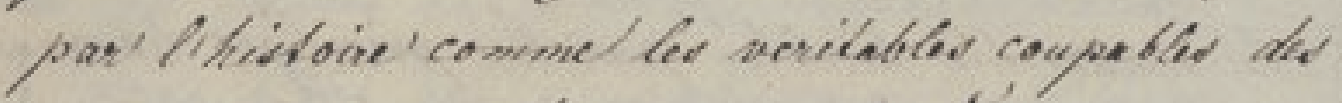

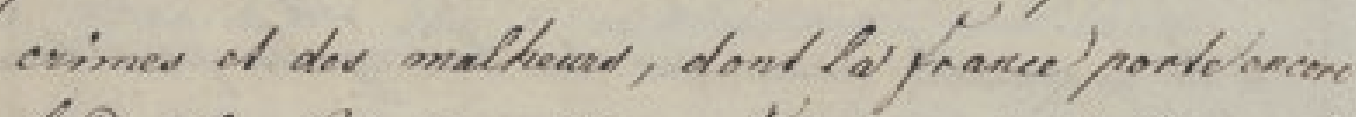

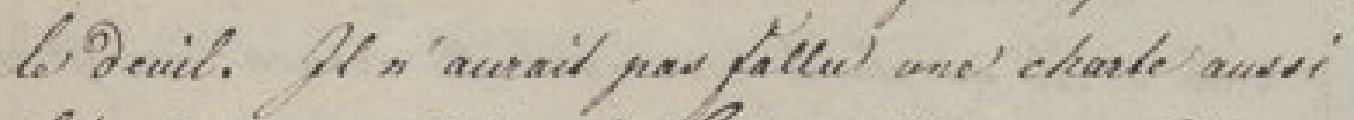
liberale' que celle' que ' 'erris XVIIT. a aceendee pan

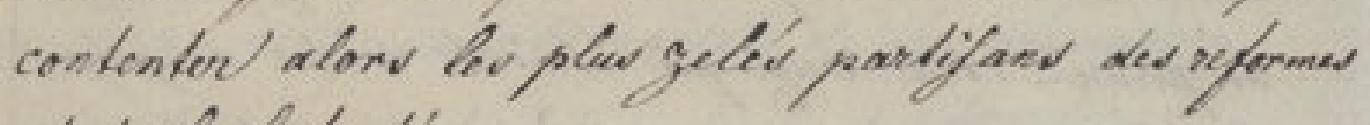
of de la liberte.

La reostulion frimendic ryant the lisconage

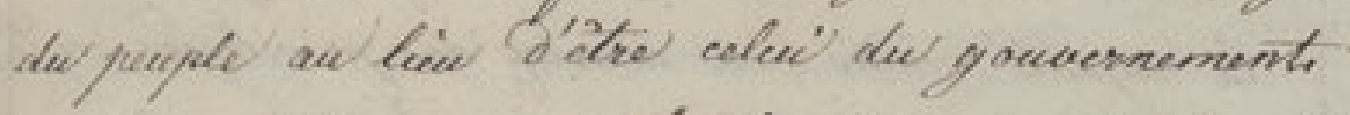

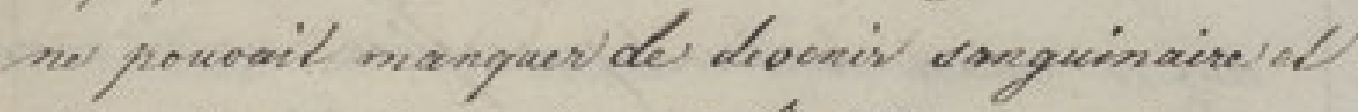
atroee - un torronl, qui frust D'wre main verou-

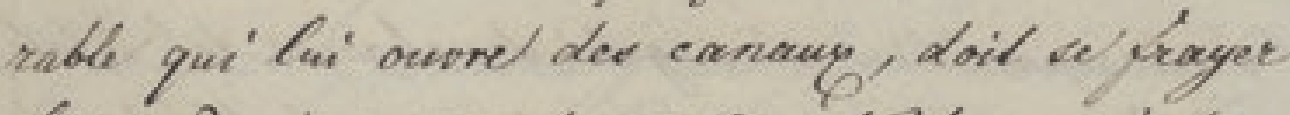

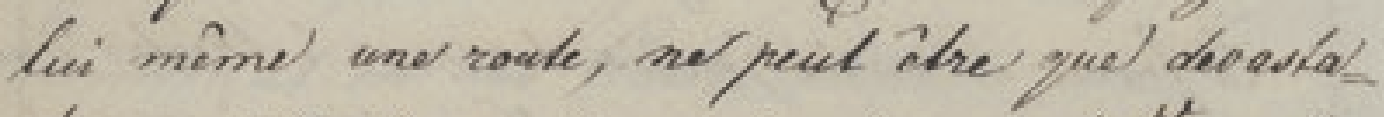
tewe, et les traces de ces degates ne's'affacent

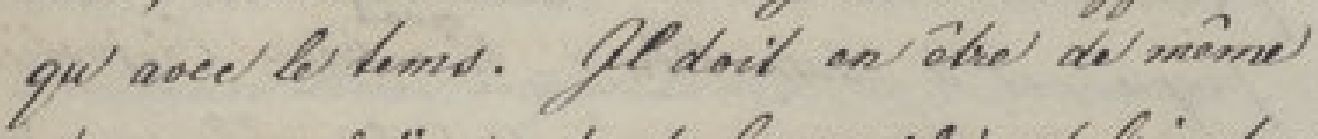

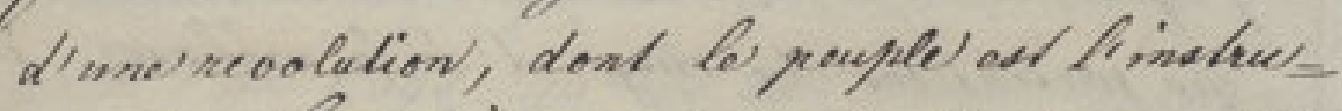
mest. Les eptrimes se touchont en morales den prolitique comme an toute chove, el c'ed ainsi opel fimnarehiel a) due necessairement che scivid dew despotisme), pascequel lal forced

dente

74 
915

sente peut methe' un prein aw decoidre of a' lietat de scoolle. On no sacenit aceuver nops

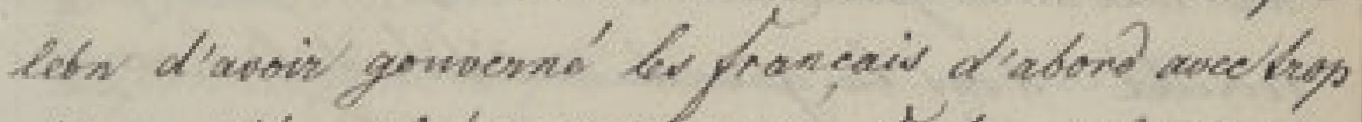

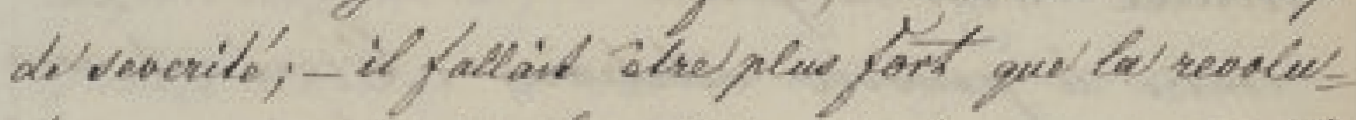

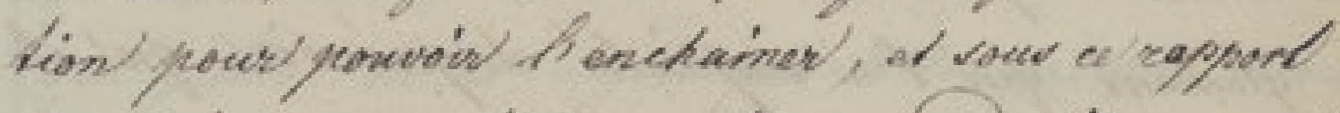
Rruobion deona toujous etre convideré comme lheros du vieide, it n'a'ea d'égal, ni on amace,

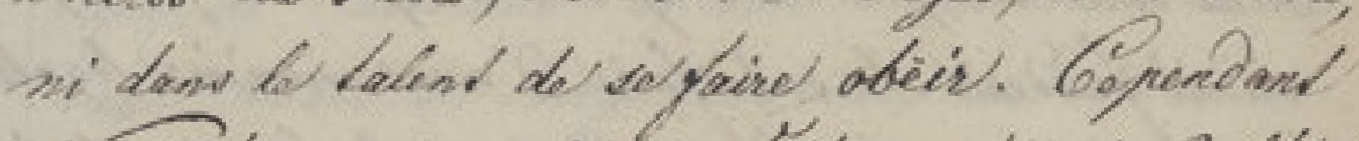

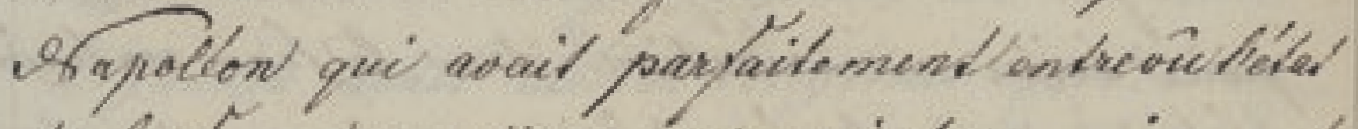
de la pronece en 1999. "I'a proint compuid dan

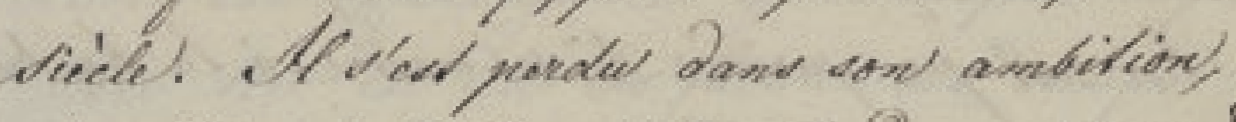

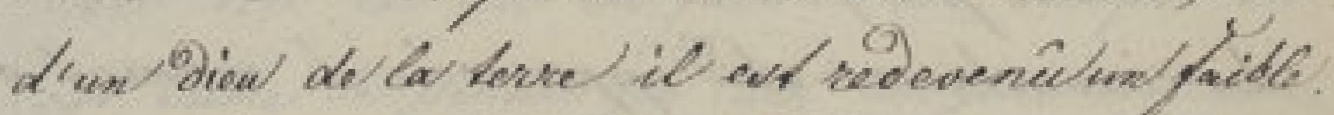

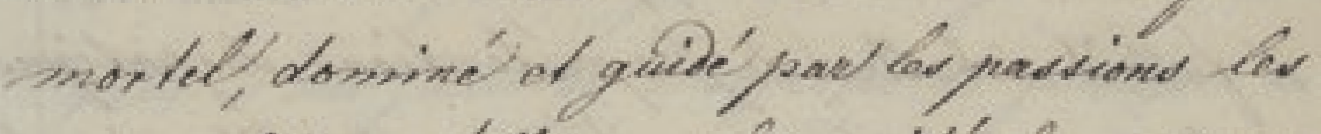

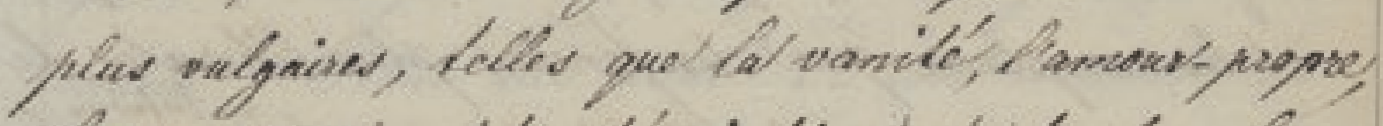

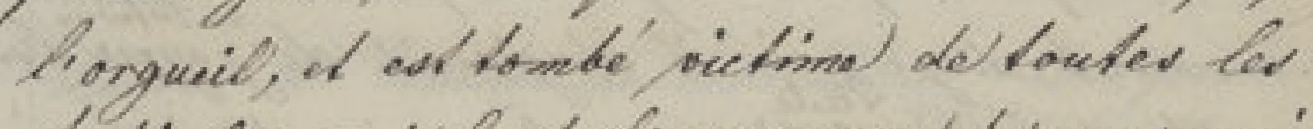
paibleses, quit fon' lapparagel del cer paves =

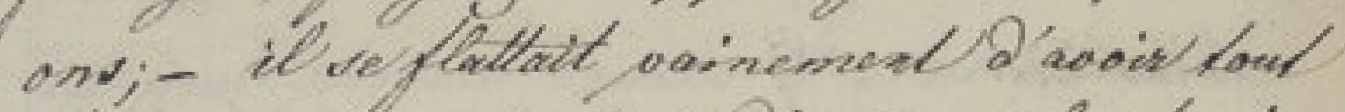
lail en placant le diadime we fon pront it de crognit tout paivant puisurit avait fii momentamement impever silencel a' Pogitation des pastis - maid il ne reyail

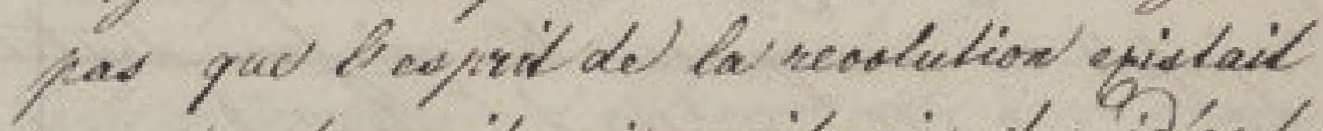

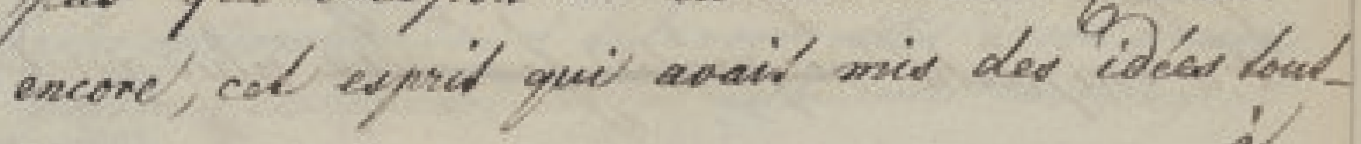

75 
16

a paif nowatter en ciculation, qui avait deja ponit

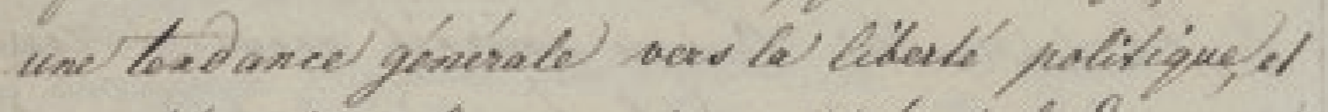

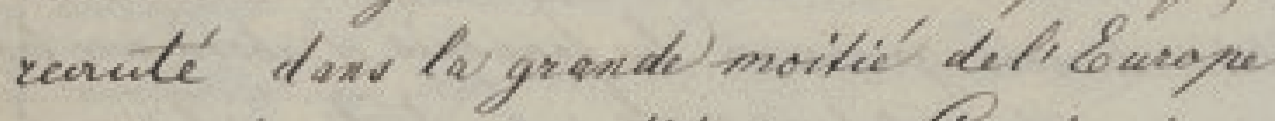

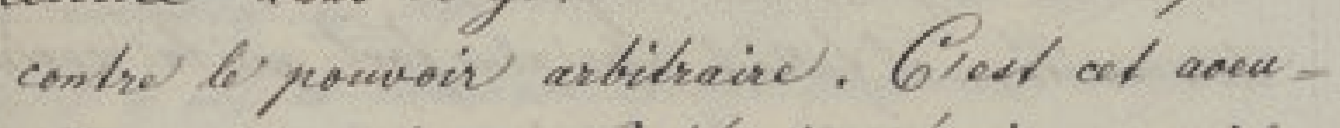

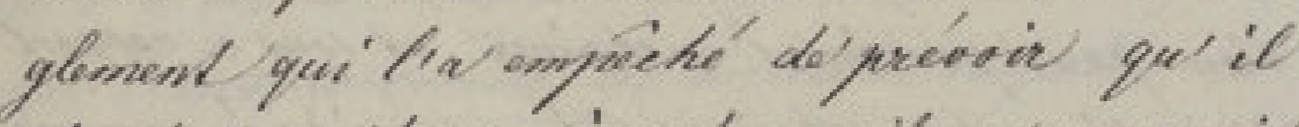
viendrail un temo, aie at exprit ne proveraik

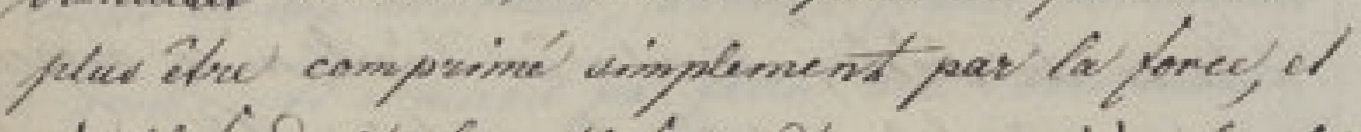
ow it facioril le salistacid 2'mo manicie beyale pour cuitur wne reaction dan befeno contraic.

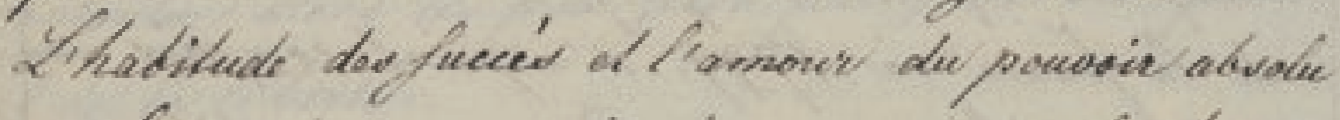

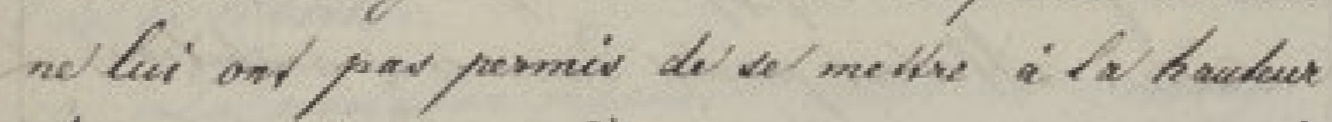
der circonstances of I'appenceosir le momene, on

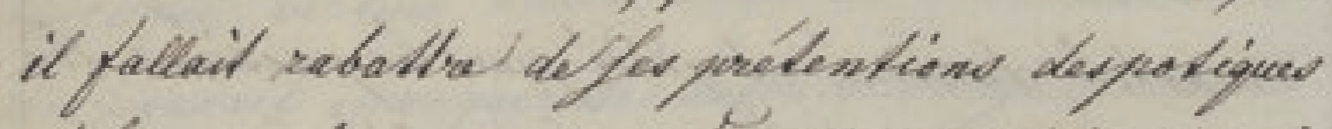
of les romplecer par des formed phes liborelas, le

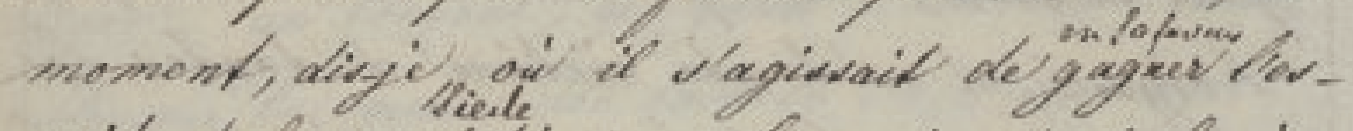

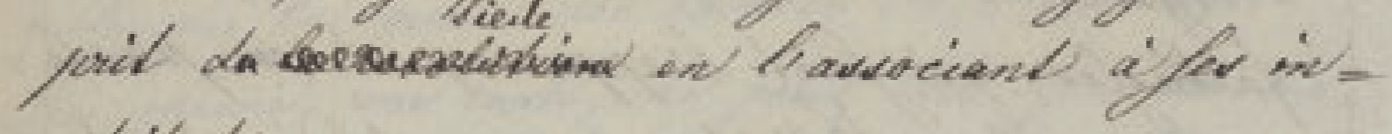
stivtions.

famais homme n'a abue' des res fourcer so pues immenfer ave moind de cal

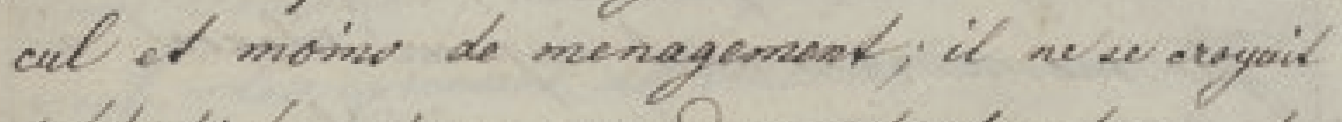
predestine' qu' aup qrander calavtrophes. agisfaid on consequarce. 6 e qui fan donte

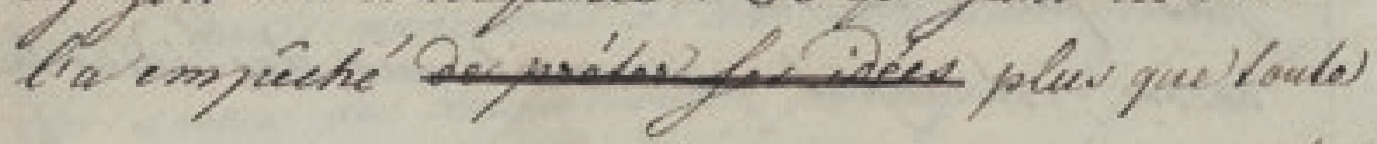
aulue

76 
1019

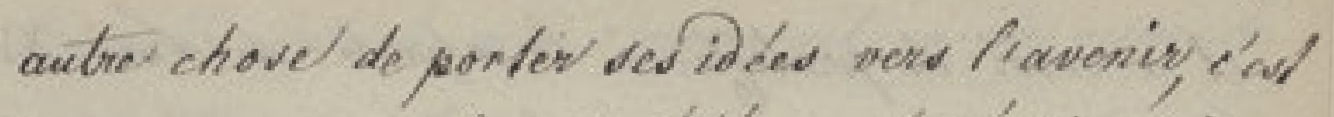

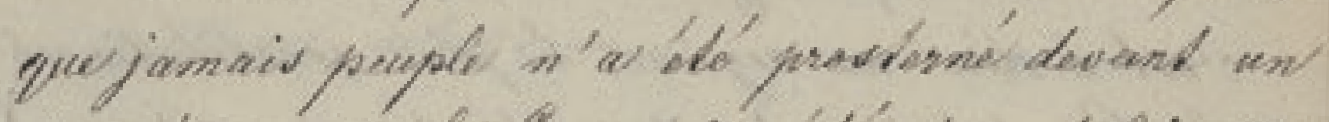
despote comme la freme pi a cle deasur leie. On'

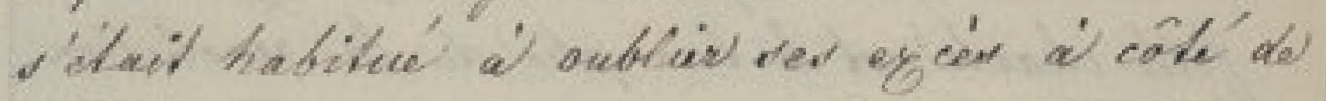

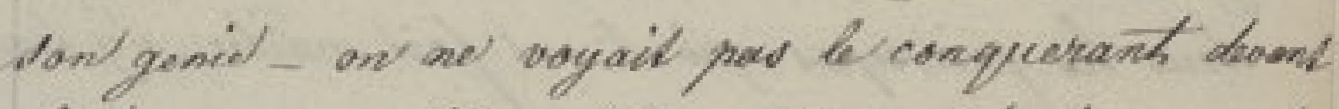

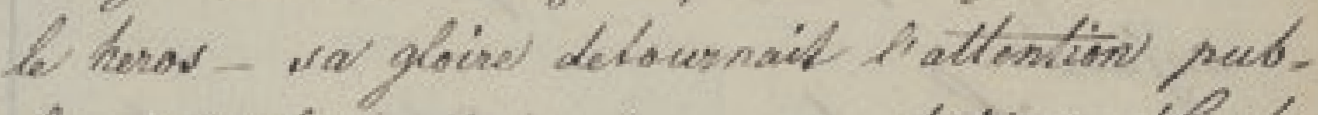
iique de fietendue de won ancition. alaf for tune sacil dilongreond veconde' con acedace

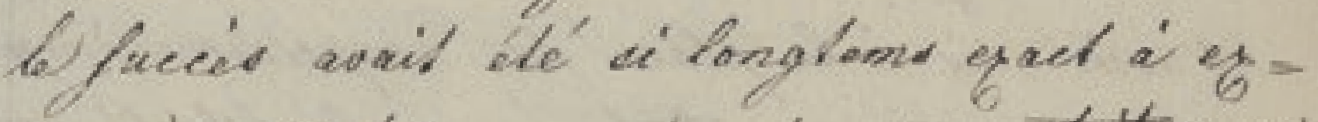

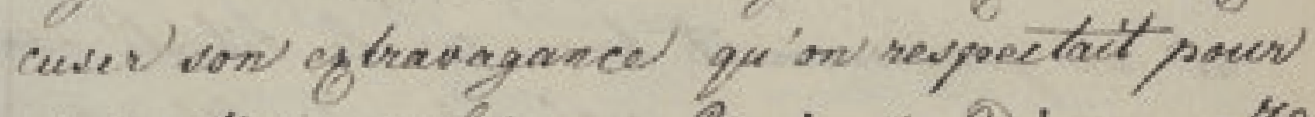
anvi dire en livi un proveri dev dieub. If ent bide de pender que lant de gunie of hast des

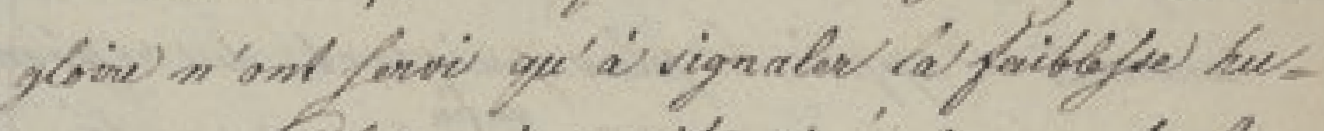
maine Colui qui soait "i sminement be noyens de beveniv le biengaimed dut genve liemasiv semble "avoid arcific un mondelentier que pour urower, comme it a tit wie meme, "qu'il "y a gu' ur' pas dw sublime au ridiucle **)

Sel perdons toudefois pas de vie que

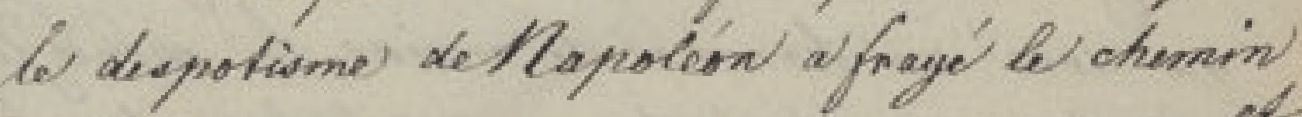

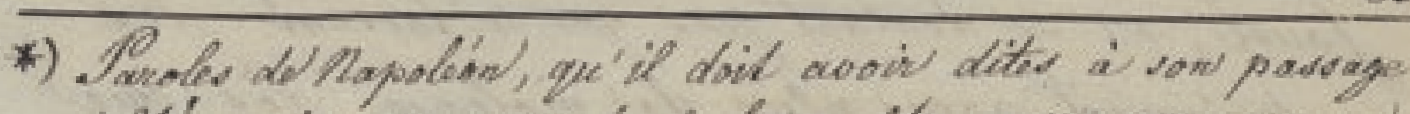

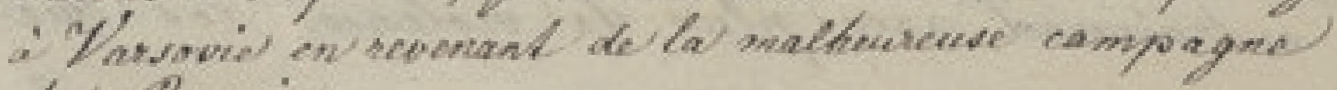
de. Ruevie.

77 
18.

ot a servi de pretefle a' la chatel de couis XVIII, c'ed a' dive an regine le plas constibutionel of a'

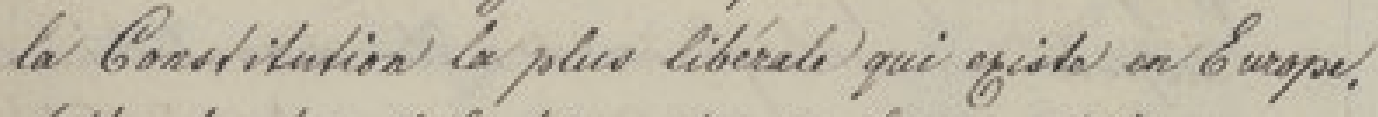
felte charte ad bi triomphe de las revolition, car tous les primcipes qui l'ont fair naitwe, d'y houvent confaeres.

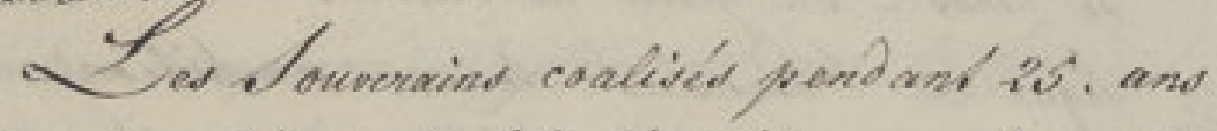
contre le systime de liberté Sitermeront un jous

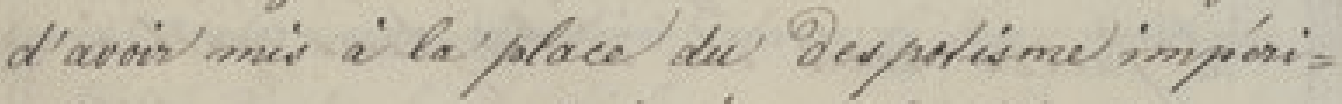
al un goumernement broe cure la liberte' consti:

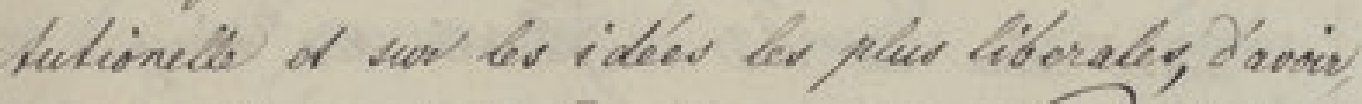
en prodamant la decheance de orapolése anemali l'appicie le plues puissant dew reqime

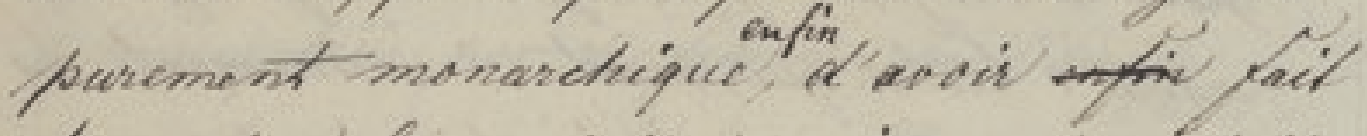
triomplese la revaludion, painemend comdattue par oup, en fridont disparaitic le feet hamme

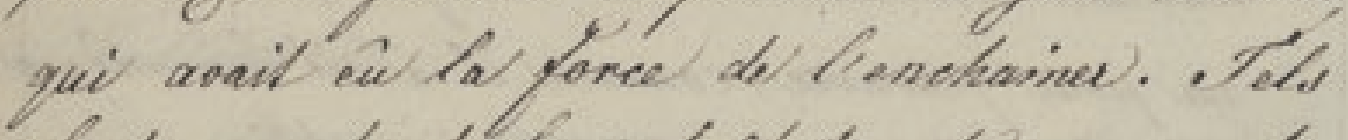
font cepondant liee nefuetales do ced vastes

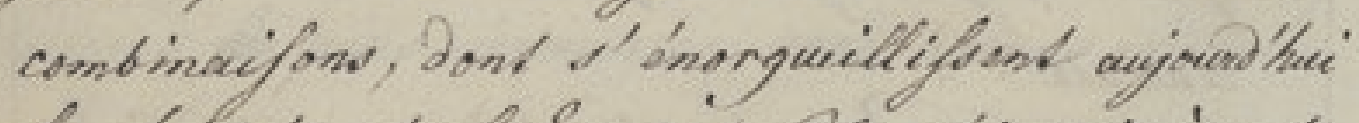

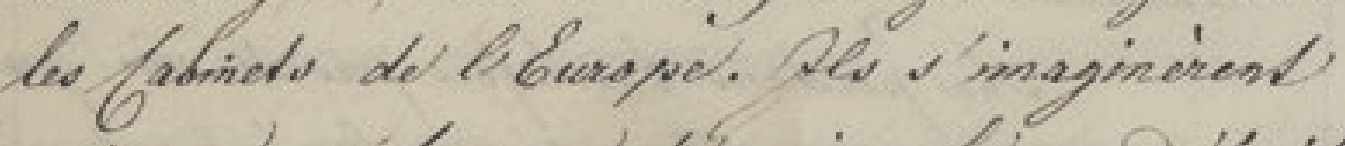
gre' aved un hommel de moine le mondefficail fause, ne se doutart pas que te torrent led entrainesait ave' plues de violenees, dis que'

le

78 


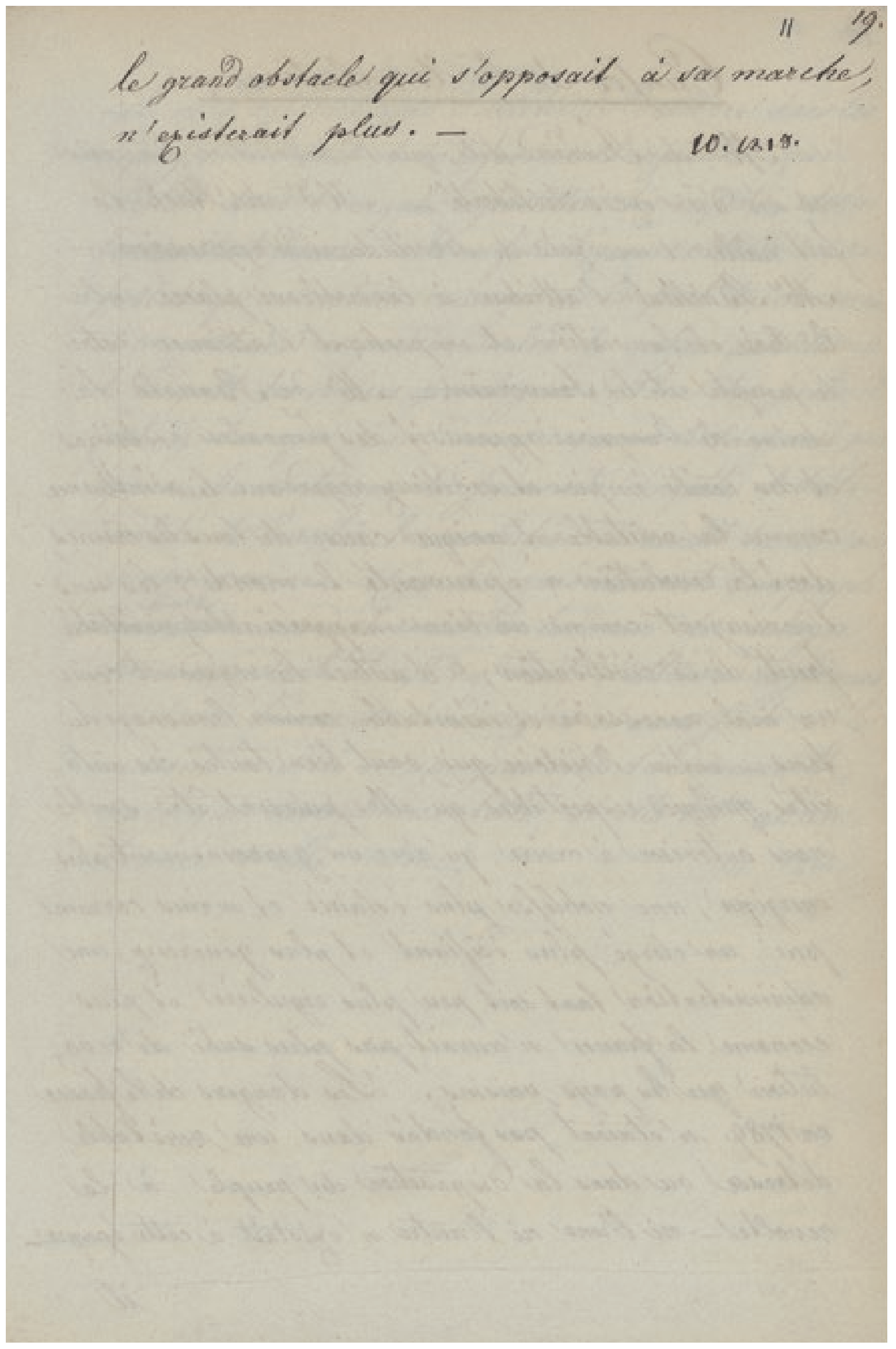


Sur Causes de la Révolution (1812) et Louis XVI (s. d.)

Ici aussi, on peut mettre en regard les textes manuscrits avec un commentaire de Johann Philipp sur ses lectures. Il dit en effet, à propos du jugement de Lorenz von Stein sur la Révolution française : " Les Allemands veulent tout rapporter à une forme systématique, y compris l'Histoire [...] La Révolution française n'était pas la conséquence d'un système philosophique - pour tout dire, elle se produisit car il ne se trouva personne pour lui montrer la porte ou la contrer avec quelques réformes raisonnables [...] Elle tira son succès de l'irrésolution et du manque de courage de Louis XVI ". Wessenberg écrit encore : "Le brave Louis était un homme bien intentionné, mais au fond un pauvre bougre qui n'a expié sa faiblesse que par sa mort en martyr ». 
20

Guifes de le kevalietion,

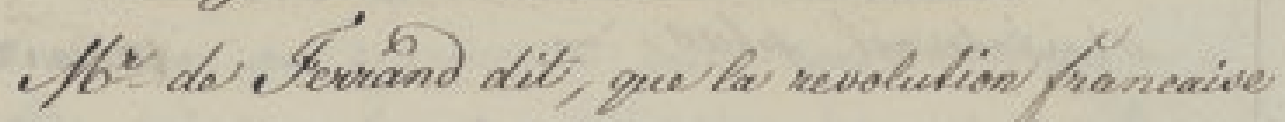

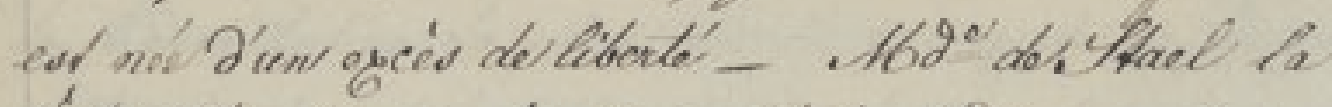

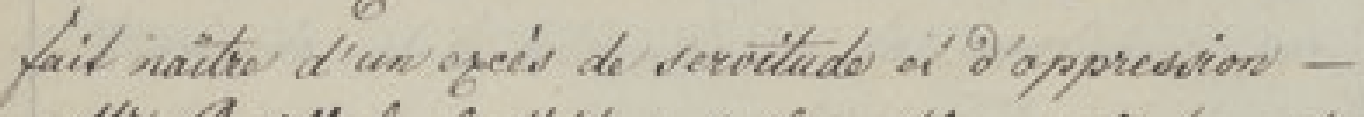

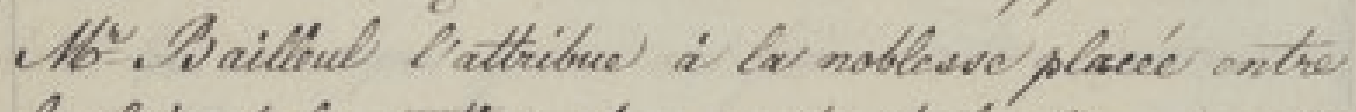
le hoi et has malion et empechant tixlianed entes

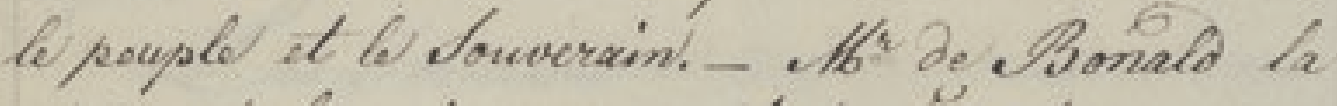

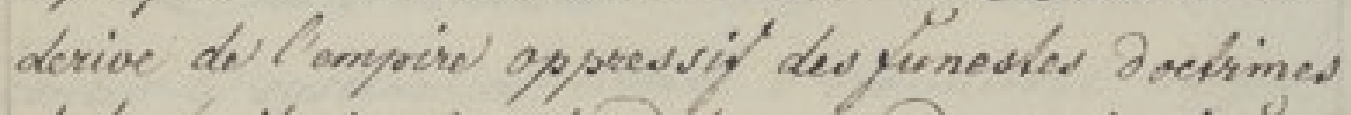
et dos ecrits impies of sedilieug regardani leuv imflusence comme la writathe of unique cauve de lous lis crimes, dan' lav undulion a éporvente' le monde; les unv lonvisagent comme un bien inapperiable, weritake frut de ca civilisation; Od'autres la regardont come un mal necessaire or inévitable, cornme fiourage du

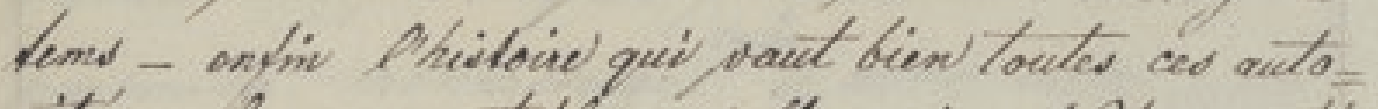

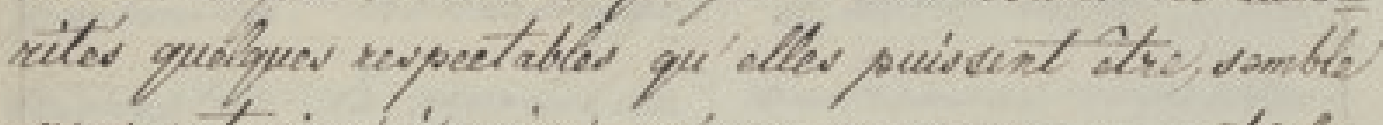
noud autoriser a'croive, qu' avec un gousernem ont slus

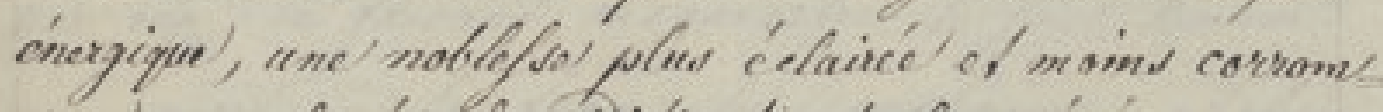

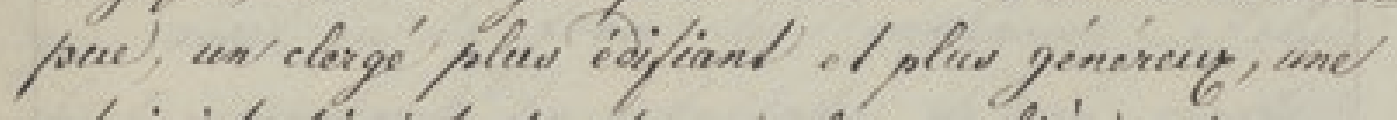

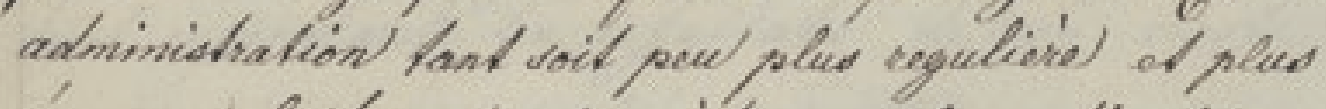

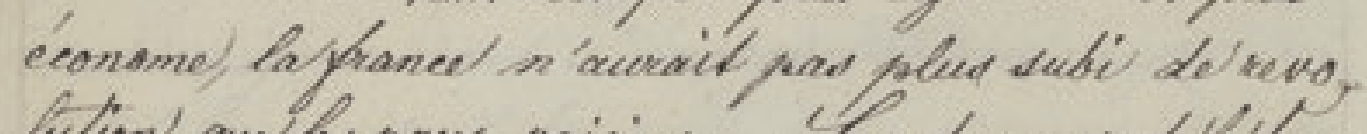
Lution que hus prys voising. Led drengers dellaphance enirgo. "s'triont pas fonde's dases une veritables detressel ow dans la disposition dew pauples is las

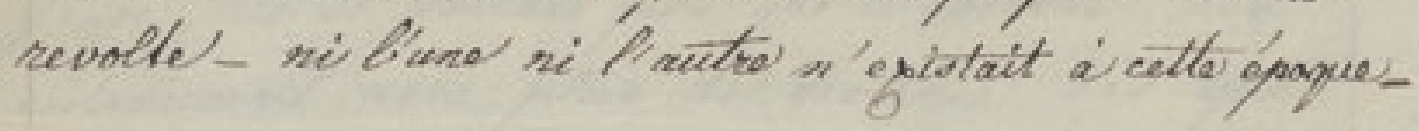

il

81 
$12 \%$

pue. dacuor lo prowa.

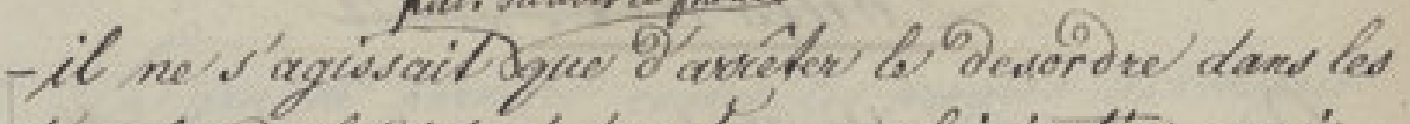
depenfes del stat is do reformes pimpietice qui axistait dand finégale repartition dew impots, butcha

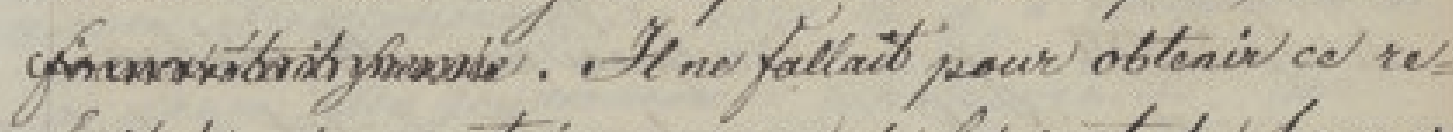
fudal gu' un cottain corizage de la' part dwe louversin

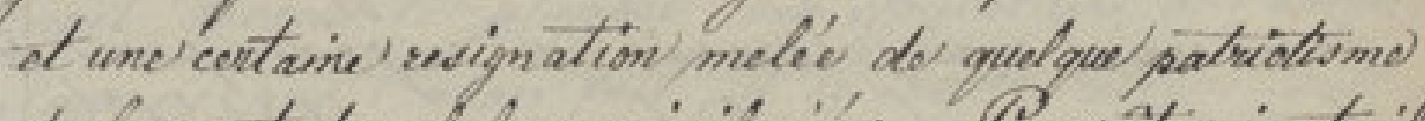
de la part des clafsed privitegied. Sour stro juste il

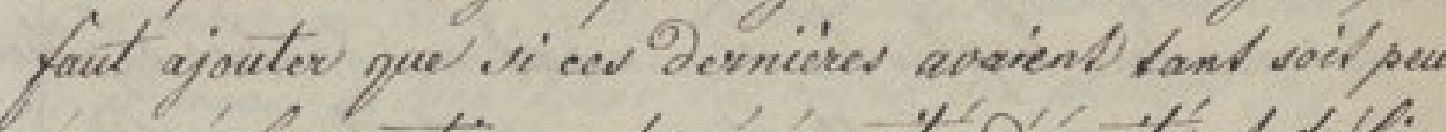

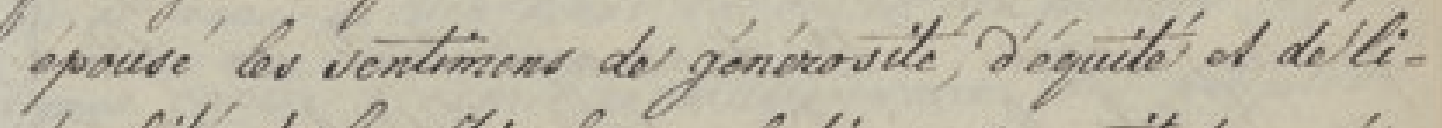

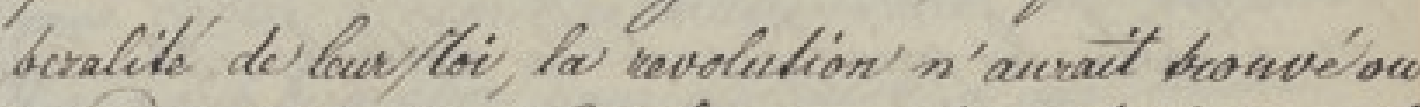
sundre racined. Gav le meeontoment om paysle numa it remontre le devir de voulager

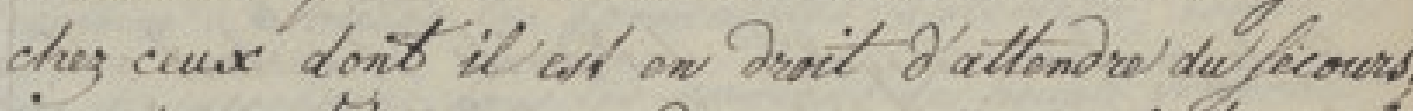

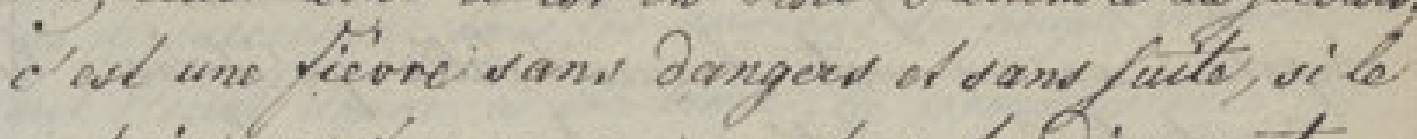
medicin ne on accupelyad trop tardivenent, mai qui devient morkelle, videred abans donnee $a$ elle meme.

11. 1816

82 
22

ouis XVI.

On n'a cessé d'attribuer une grande partio des dewasters de la revolution à la friblesed du caractire de Louis XVI. Te ne disculperai pas celle priblesse qusigu'de ail 'te' on qrande partie lieffet de la bante' de son coever; maís 'obsevertai quel ce pince pour we,

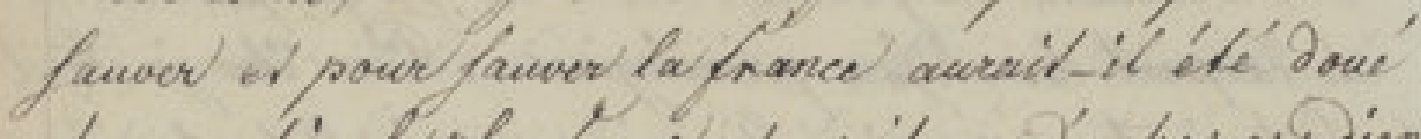
du caractere lo ples fasme n' autail see d'autres expedions que les fuivans: 10 de renooyer tante wa Gour,

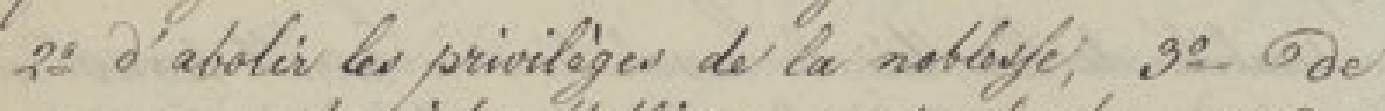
supprimer las richos stablissesnens dev clorgé, 40 de

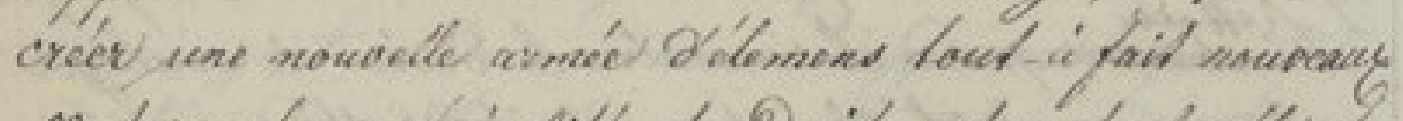

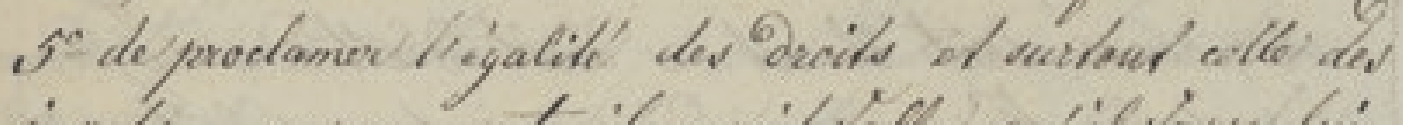

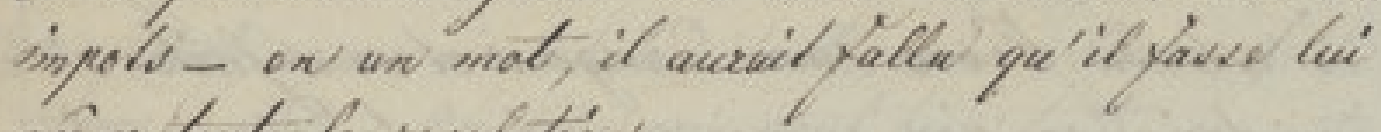
mecme toule la revalutión.

83 


\section{Sur Napoléon (1814), les Autres réflexions sur Napoléon (1806) et sur La France (s. d.)}

Les vues de Wessenberg reposaient à plus d'un égard sur ses relations sociales et mondaines. Il recourt ainsi volontiers à Mme de Staël : " Elle a dit avec raison que les Bourbons auraient dû se contenter de s'asseoir sur le siège de Napoléon, plutôt que de le reléguer dans les mansardes et parmi les vieux meubles ". À propos de l'histoire de la France, Johann Philipp, ambassadeur à Kassel (Électorat de Hesse-Kassel) en 1806, constate : " Elle est à cette époque un drame incommensurable, conçu par la soif du pouvoir, mais mené par des héros " (les troupes de Napoléon occupaient la Hesse électorale en novembre de cette même année). "Son cours politique inspire souvent l'effroi, son cours militaire presque toujours l'admiration".

Peter Heinrich von Wessenberg

(traduction Christophe Didier)

NB : certaines pages blanches du manuscrit n'ont pas été reproduites ici, entraînant çà et là une rupture dans la numérotation des pages. 
- Sapoleón.

13 29.

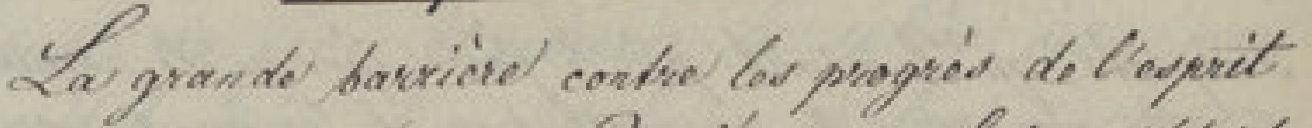

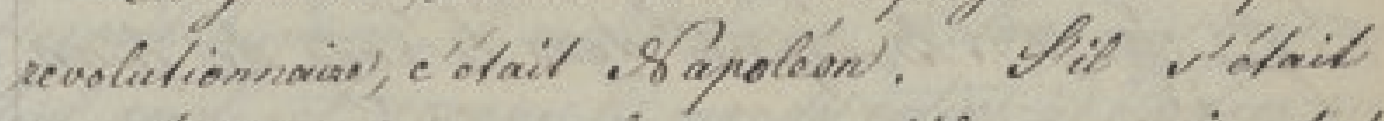

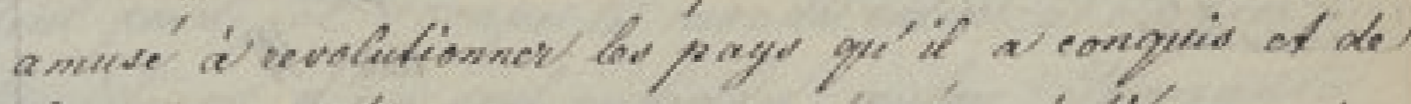
Lues dosnes des goweonemene copresentalifs, qued

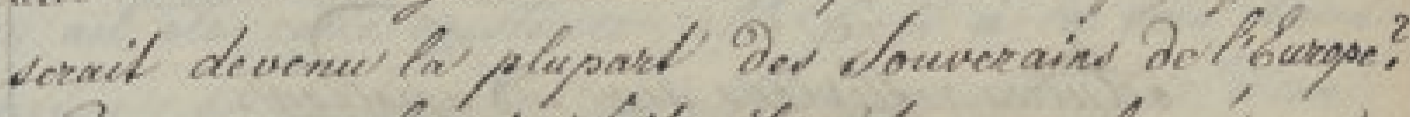

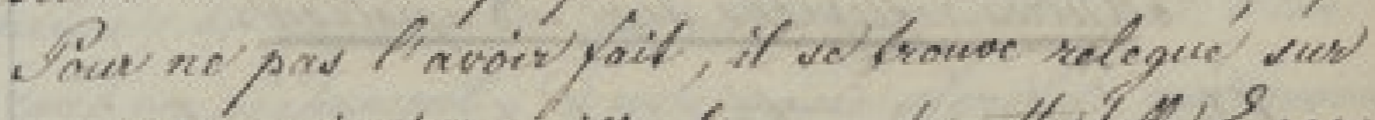

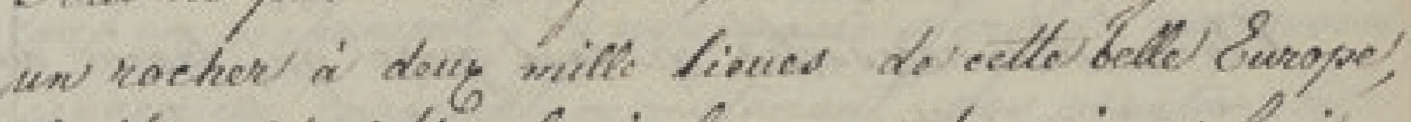
oi 'it avait si bien foroi la enerve des primeed logie = limes contre (esperit reaslutionnaicel des pereples.

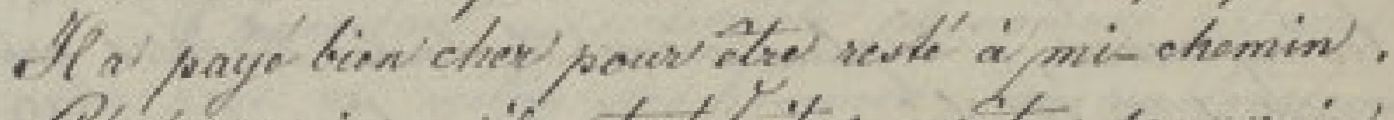

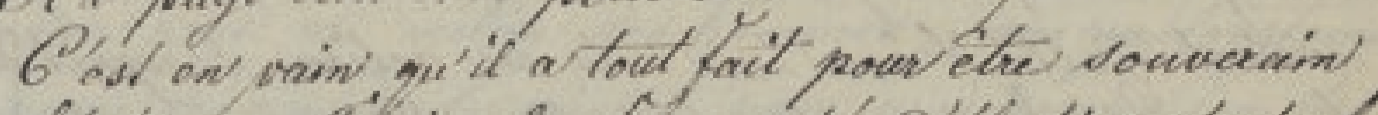

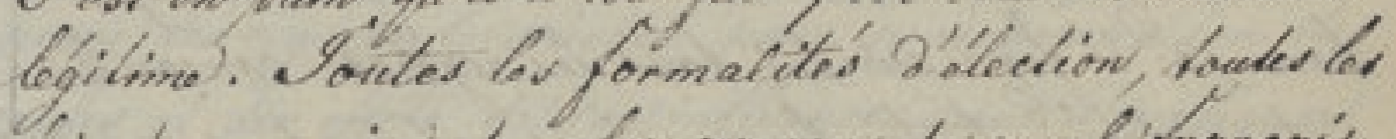

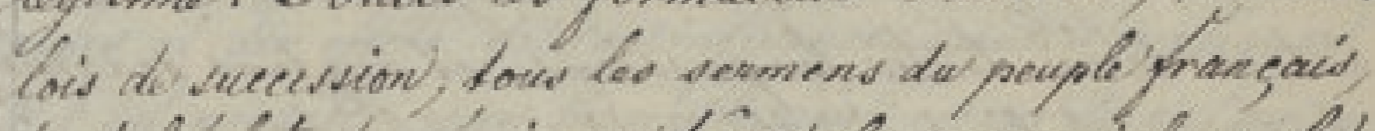

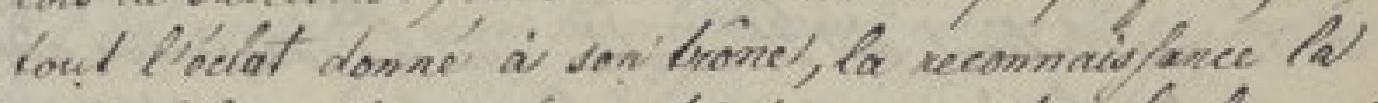

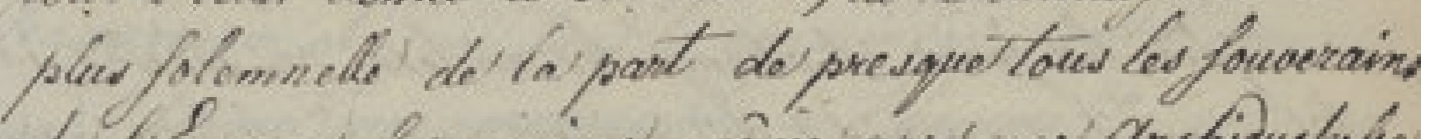

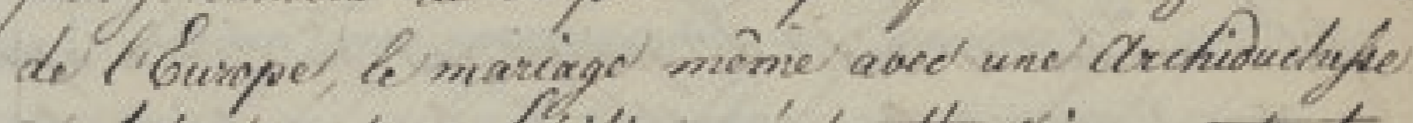

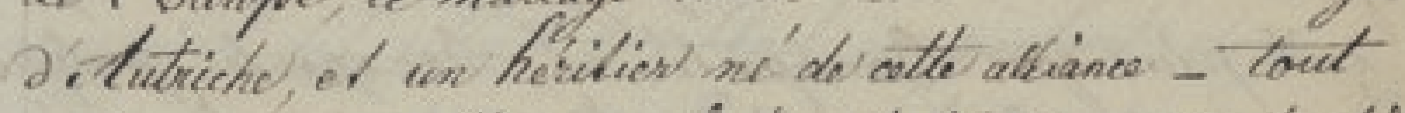

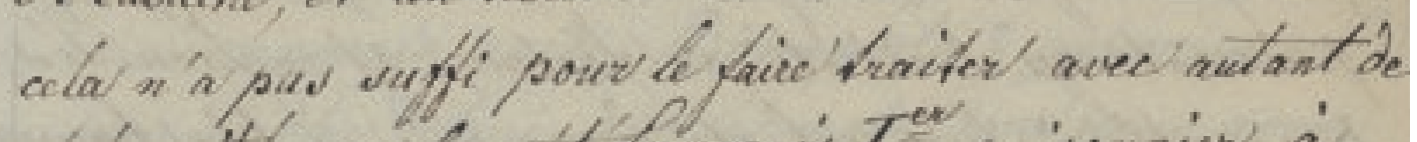

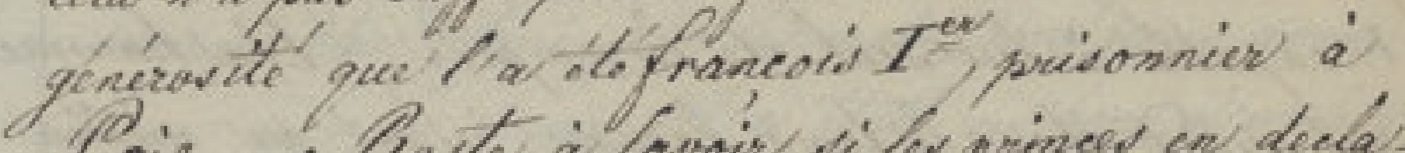

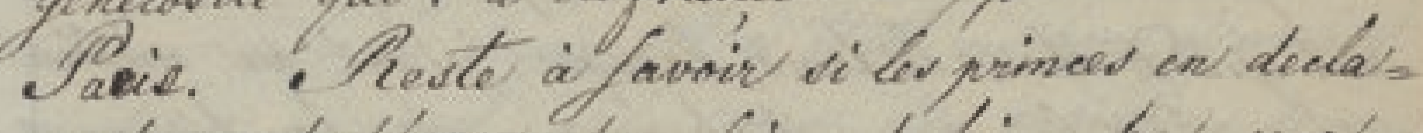
ran. In detéance ef en laivant triompher une

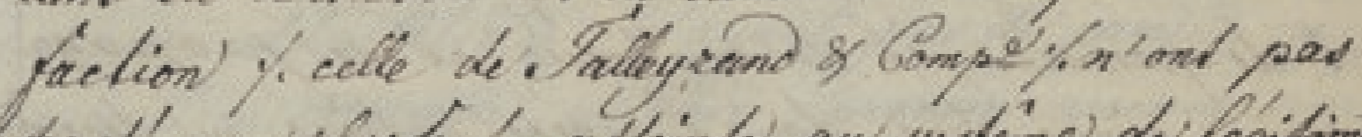

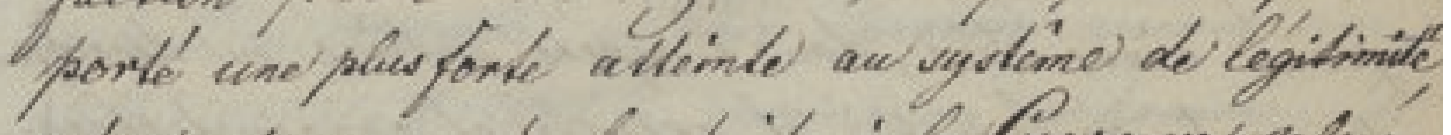

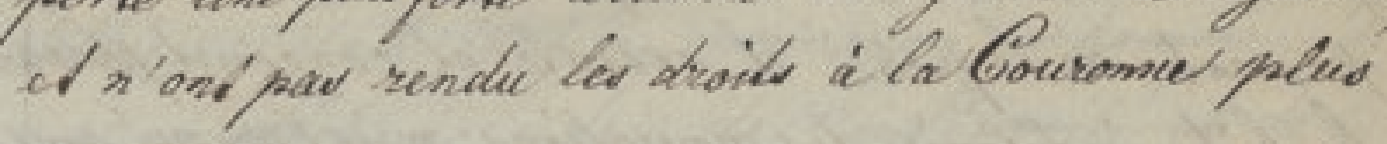
sers =

85 
$2 / 4$.

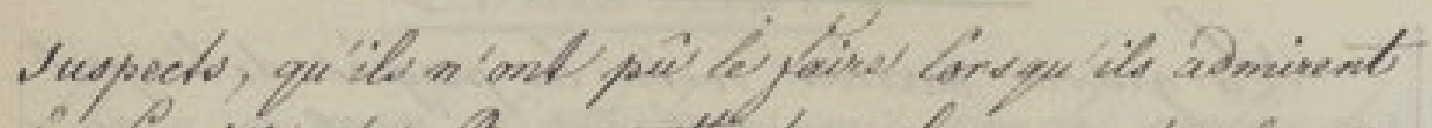
la pamili de. Bonapate dare le rang de lowed ginavies.

1814 IV.

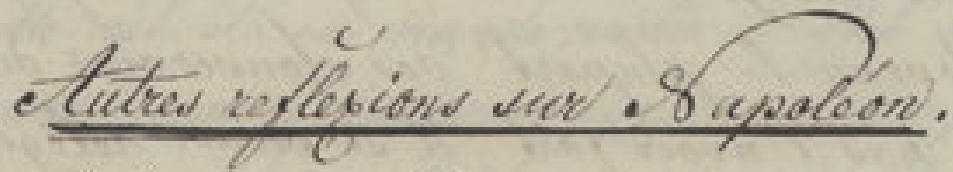

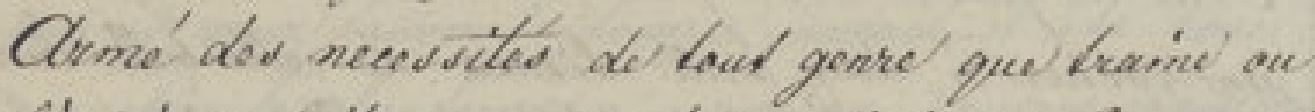
fouleve á va futto une grande zeadedion Bonaparté

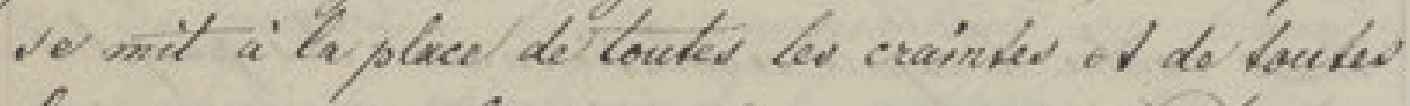

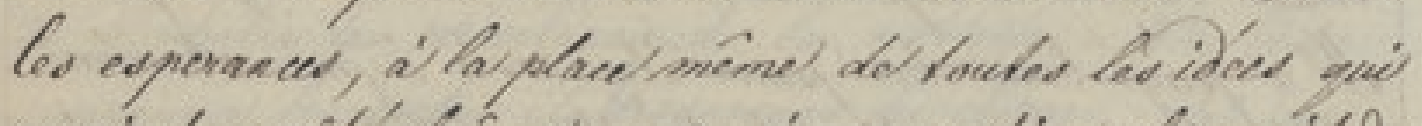
avaind ecalte linme ond mes en action lespit das

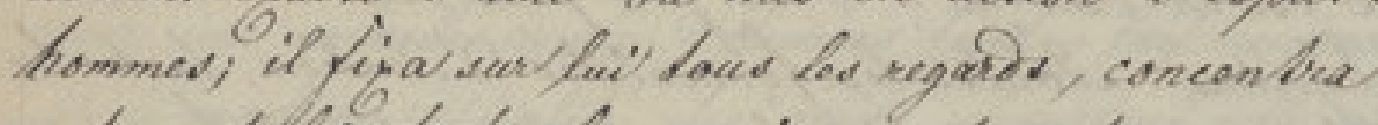

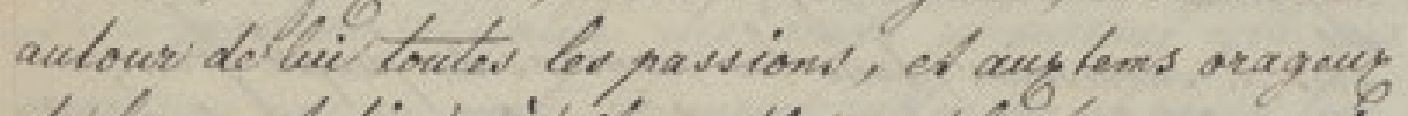
de la rearlution, aid it somble que led evensmens aions

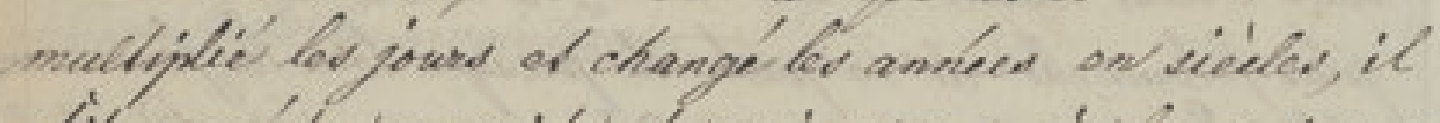
fil eneceder ce videl de quinge ans die ton n' a pers coit plur que Bonapaste, of Bonasaste, qualou

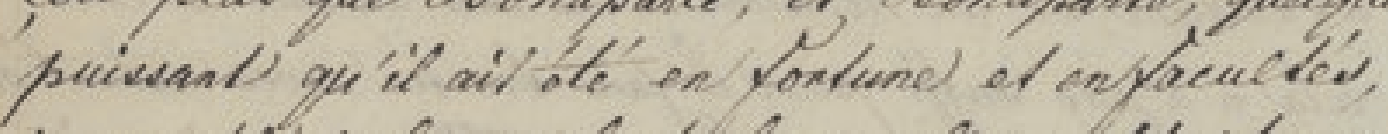
ineapable a' bei veal de levemplis. Un homme

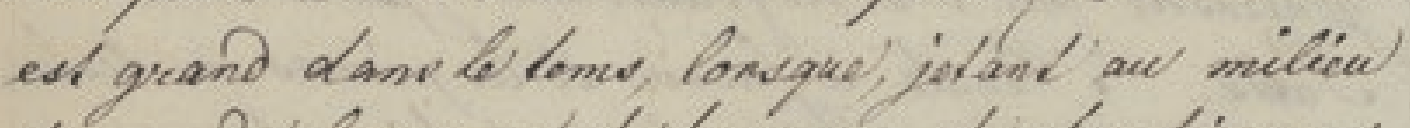
du monde be gesme de beauroup, de. Ientimend et de penved il de continiee et e prapaga,

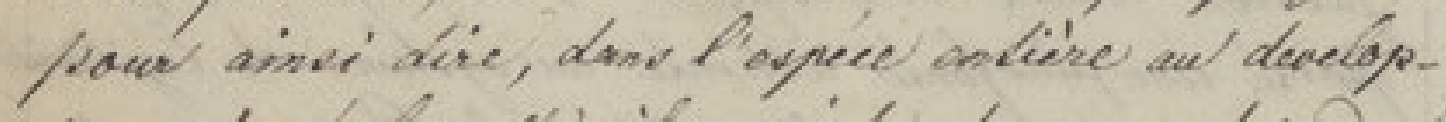
pement de raquele il aeviste of concout pendane

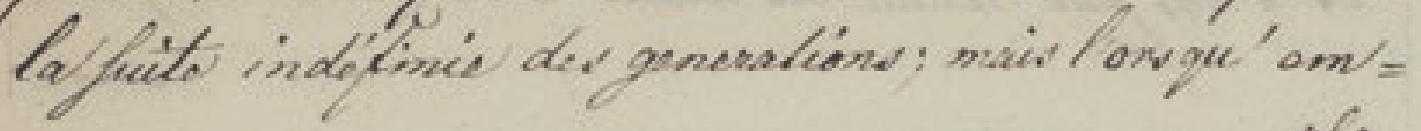
nes

86 
14. 2.5.

ployant sa force à reprecir la sphare dio so mont feeprit humein, it ne hie laised J'han quel werd un

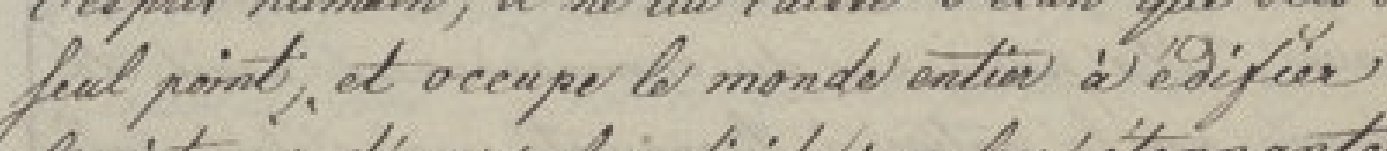

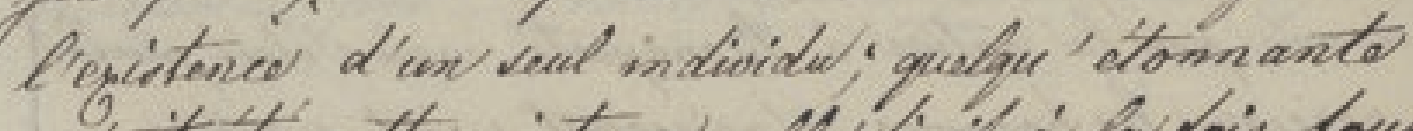

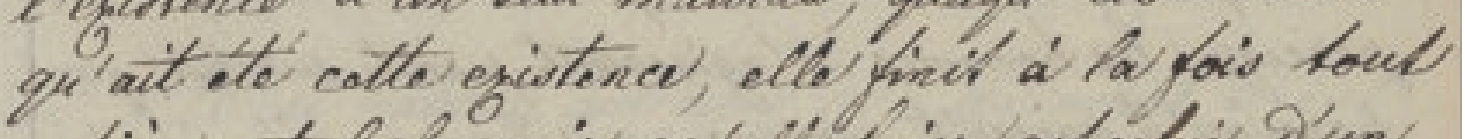

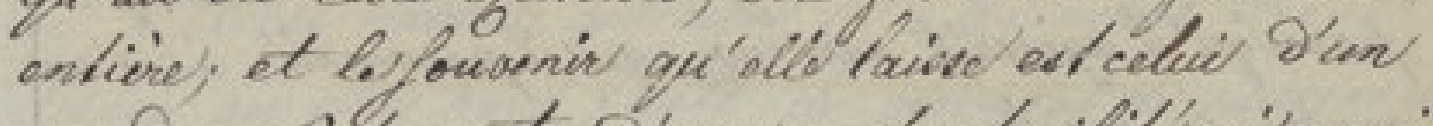

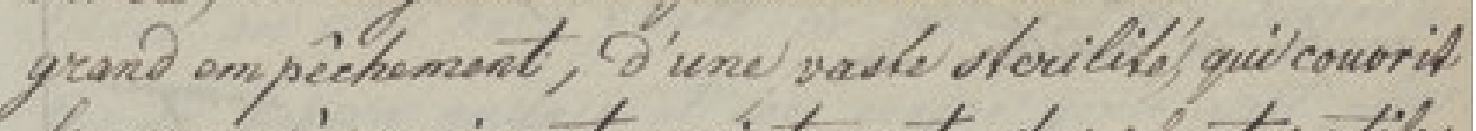
Pespace oi croissont onsintonant des prantes ntiles es lecondes.

Gependant un si terrible phönomene nepseut avoit prowe we milieu ded hommed vans que longtems

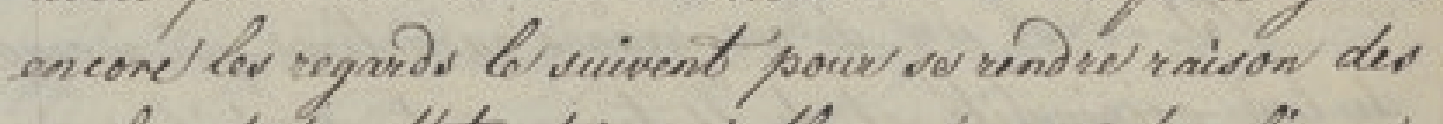

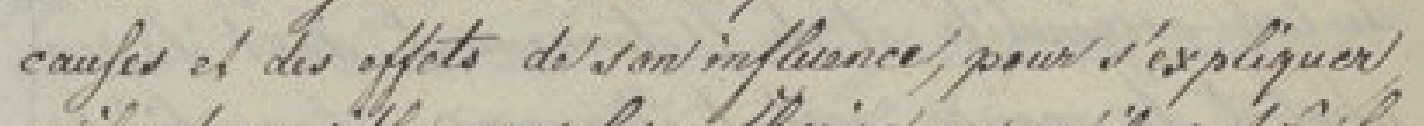

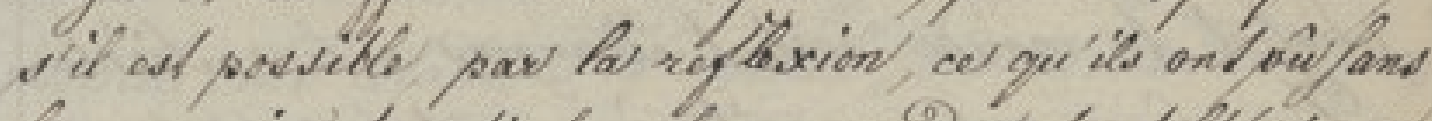

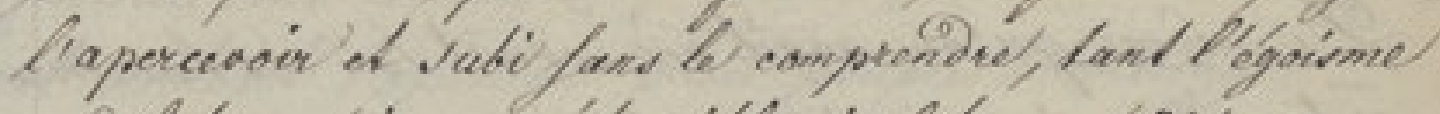

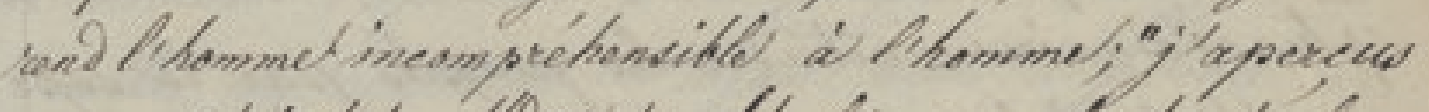
ares vide" dil. Wome de. Heab en parlant de fer premies rapponts aved Bonaparte, quelson caractice ne porenie stro define par los moks, dond nous avond

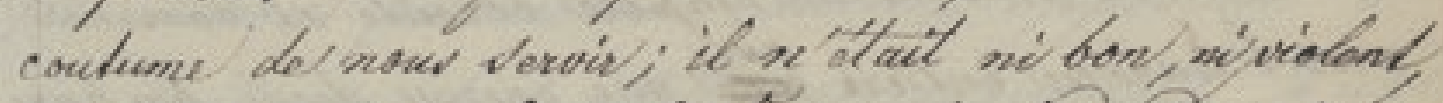
midoux, ni cruel, à la facen des individes à noses connus. Un teletre, "xyant pas de prail, ne

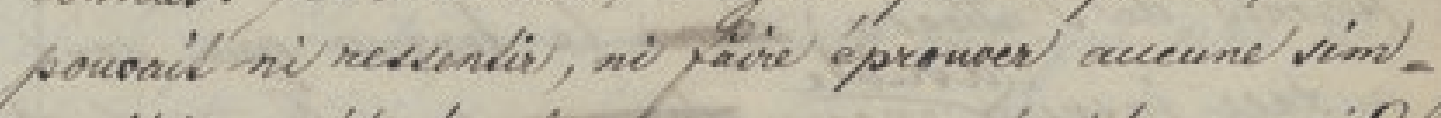
sathie; c strit suw ow moin qu un homme. It

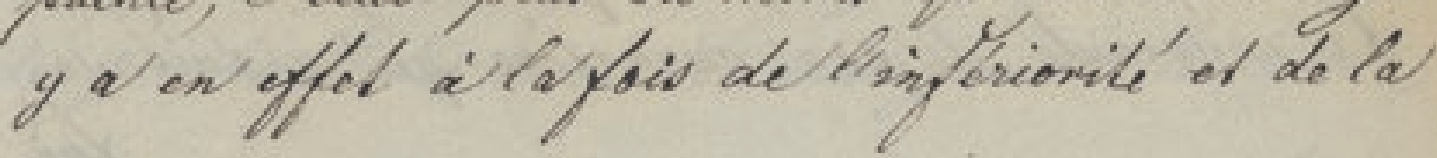

87 
26.

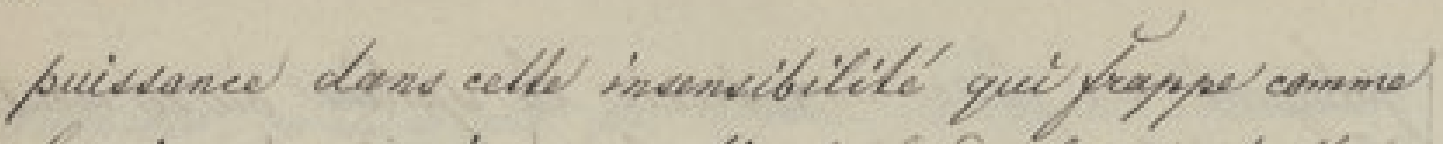

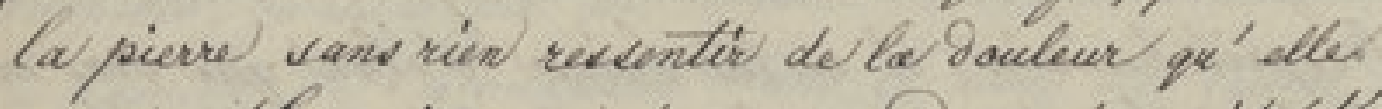
cause. La puiseance dev commandemend avxit óto'

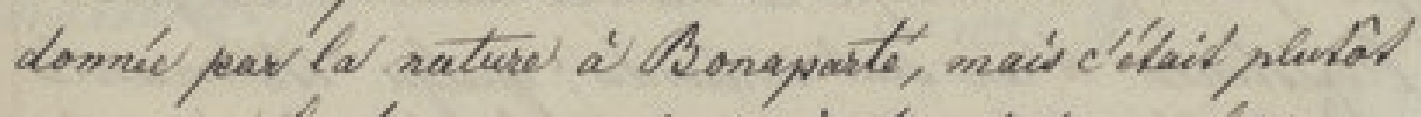
parceque las hommes in agissaiont point sur bie que

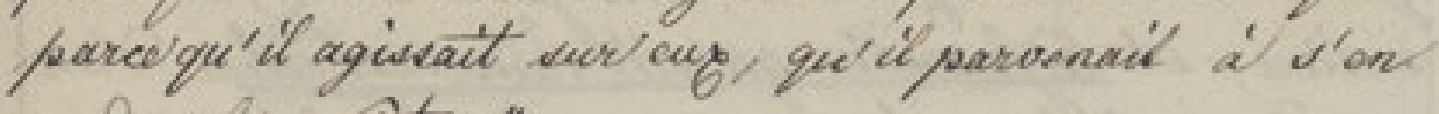
rendre le maitre." -

B... ast un de ced quander caractetred qui à canfe de bue originatile' ot de listormement, dont ilo rem plisfint ie monde, sustent itrengerd à luer liéde. Qu menee qu'on aciait vie arant lui des hommes cptraondinaires maitriser les preuples par la dupé rionité des belons ow de la bravous nowes magoned maintenont un homme sonte du néant s'elever

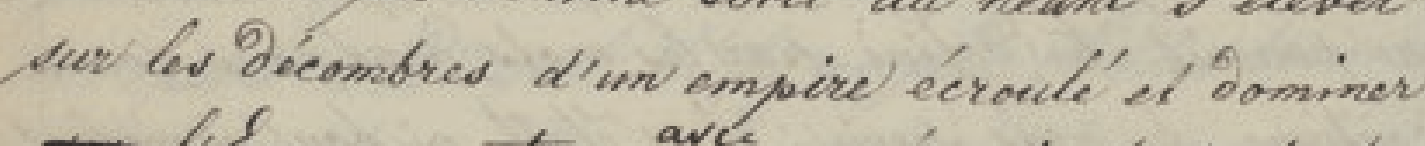
licurope ontiere ave une énergie demi-brevbar, à laquetie la civilisation de notre dicle n'ruaitpoint

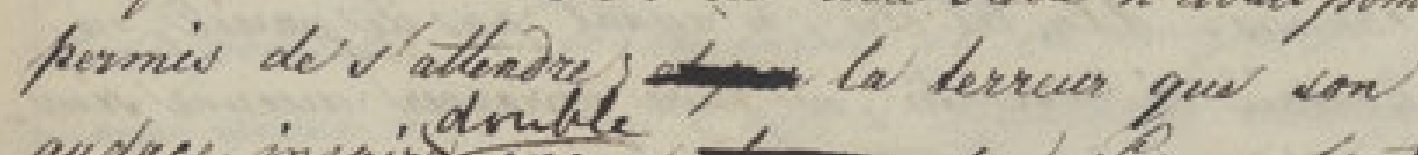

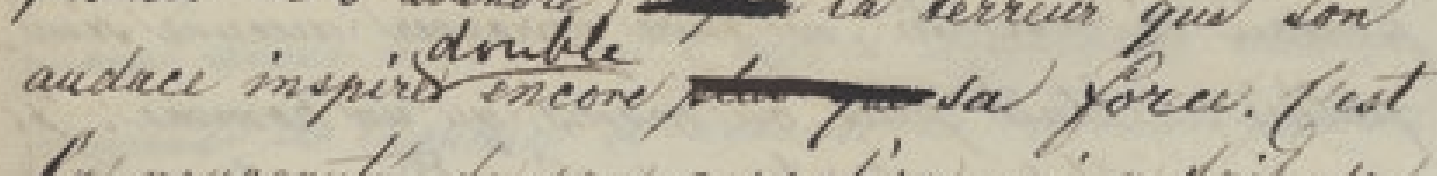
Id nenocanté de con caractire quie a ginit sa

yox

88 
$152 \%$

fortune - on "y eroyat paw on ne !y allondrit

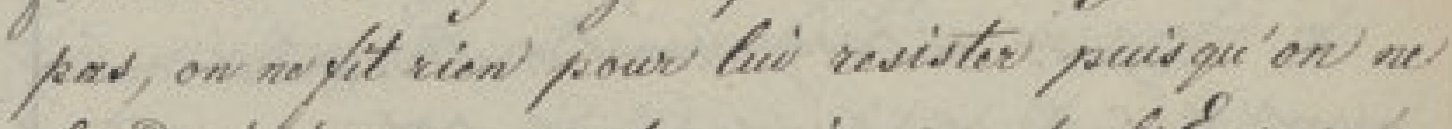

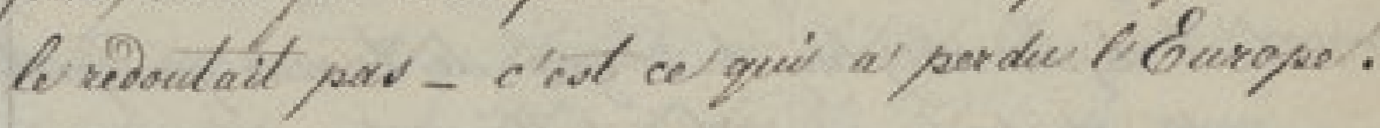

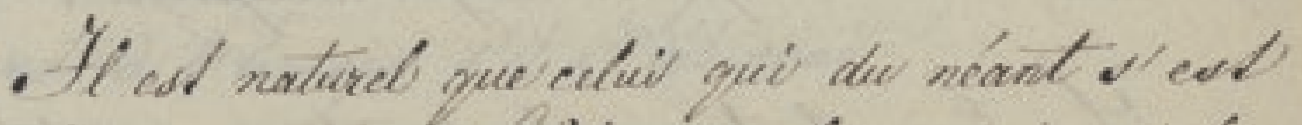

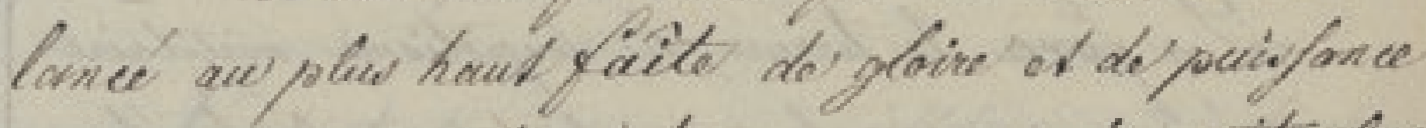

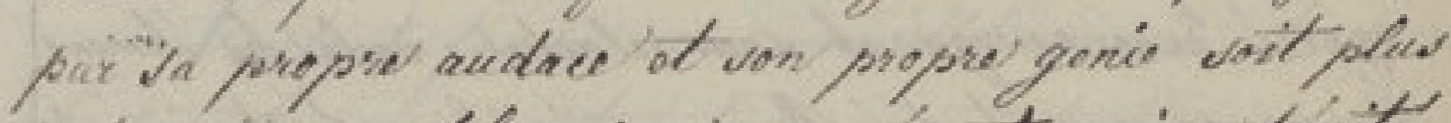
sudentable nue tol prine conquerant qui a licerte lee premioss demens de won pocuocit de fes ancetres st qui n a besain que de queloue tatent at de que laves

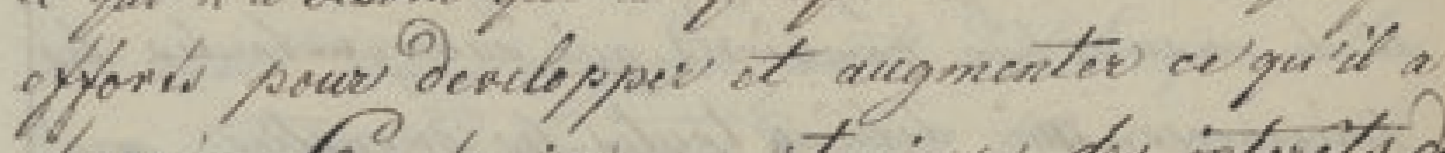

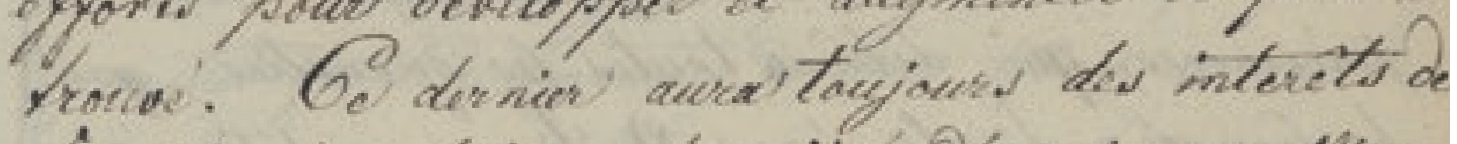
fomille, dev relitions dimitie, 2 ancionnes adiancy et surtout (apinion g'un peuple à menager -

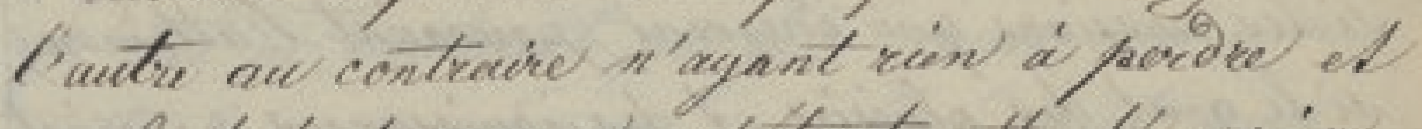

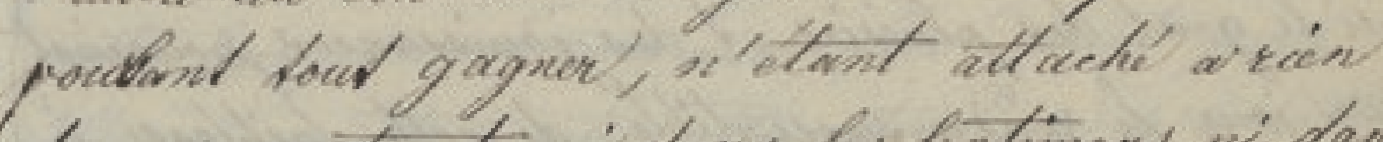
't we remonteont ni daxe fis fentimenos ni dans fro retations particuliers neceme cpposition a'

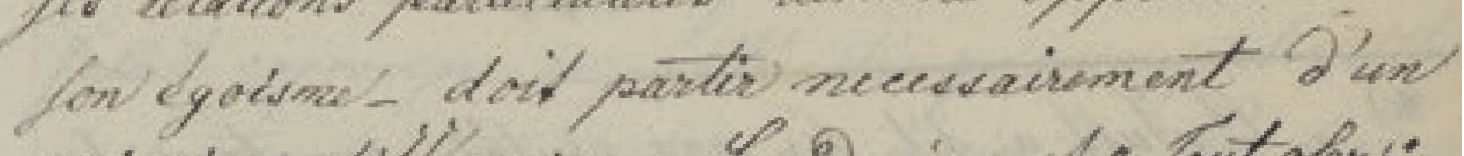

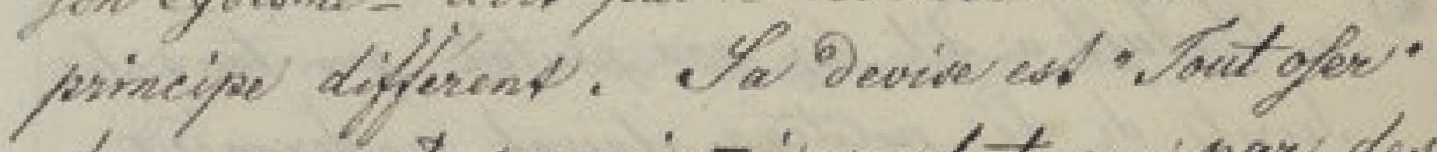
of ne powiant parvenir $a^{\prime}$ won bet que por des

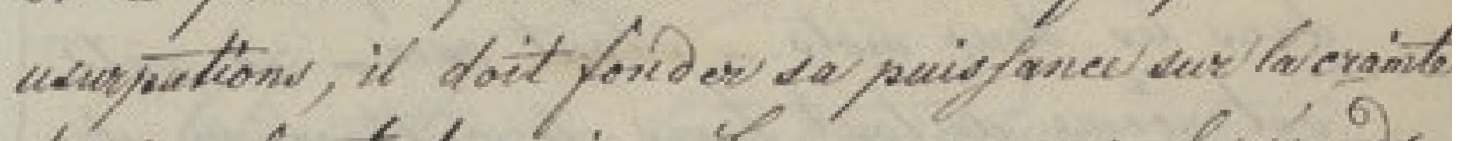

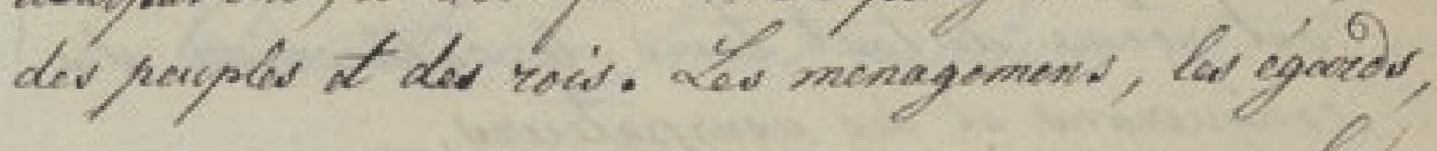

89 
28

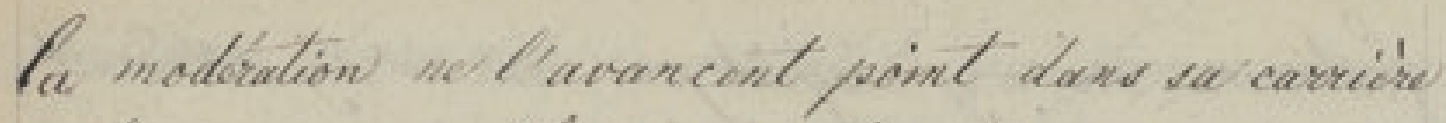

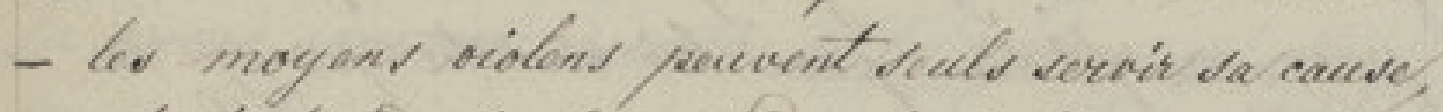

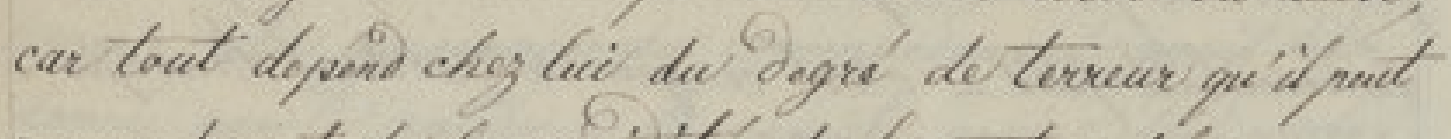
repandre, et de la rapidite de feo ontreprifes.

Ca poree la tiruer, le depsotisme mititaire

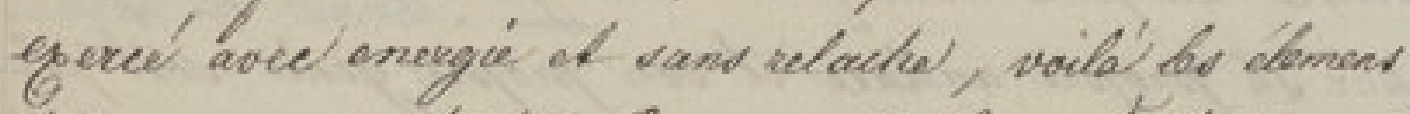

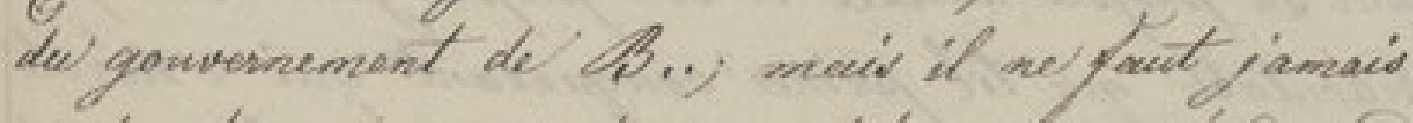

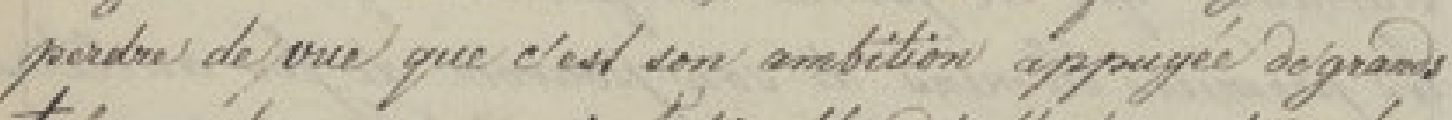

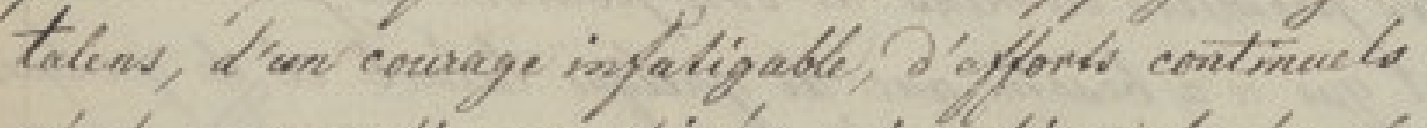
$\because$ de meveres bien combinees qui subjinguatardes bs

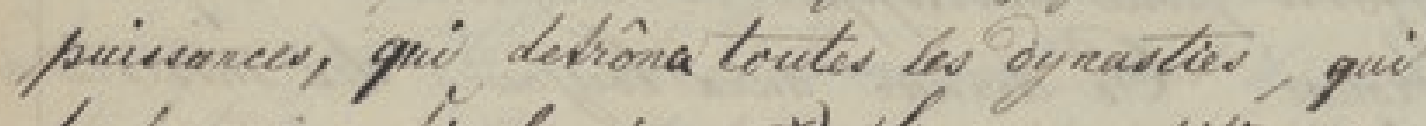

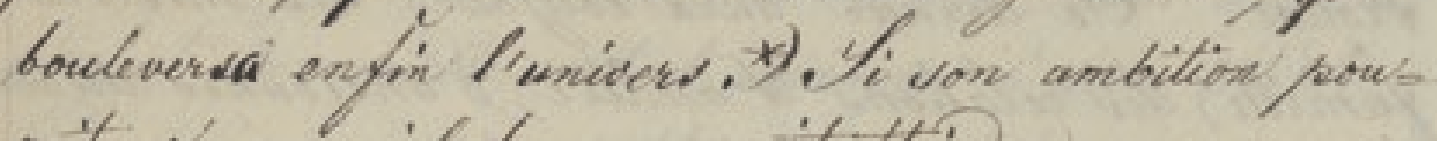

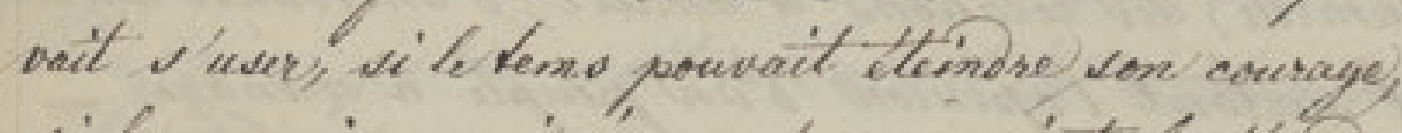

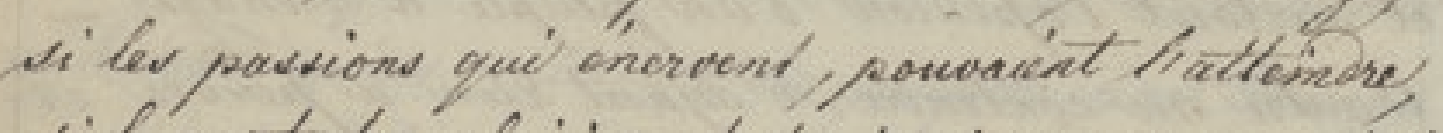

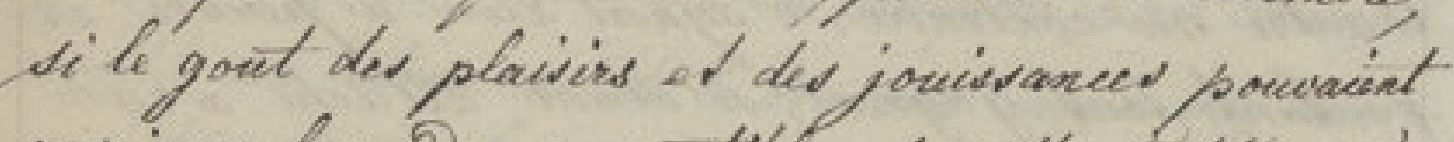
un jour le rendre susceptible de colle faiblese, a' la qualle new do herod ant cohappés. - Mlacis.

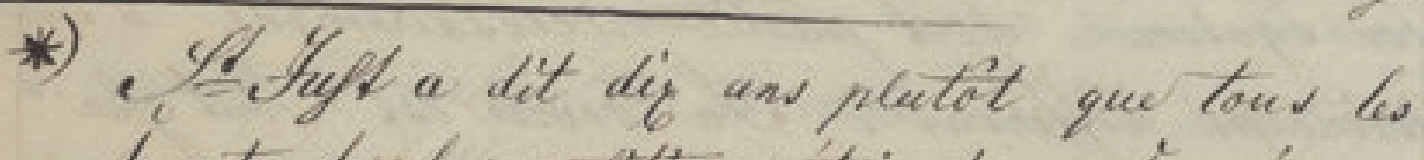
Seerets de hla politique dacint envermeis dans lev deuf mots - Dont aver-ces mots ant yorme de tout tems be cathecrions des conquerand of dev uverpatecerd.

90 
1629

i. je be dis non fane un certain cyired:. celle provece

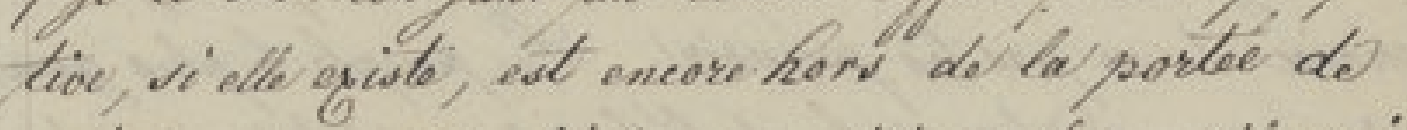
notre one. Uln italion a dit de liei qu' 'i" anail lechacas dans la tete it Singer dario le cacur qare 'a liburope dil a dit wrai.

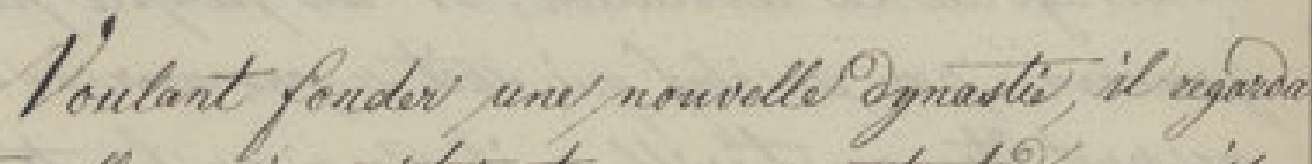
toules alles quie cxistacient comme rectant dennemis.

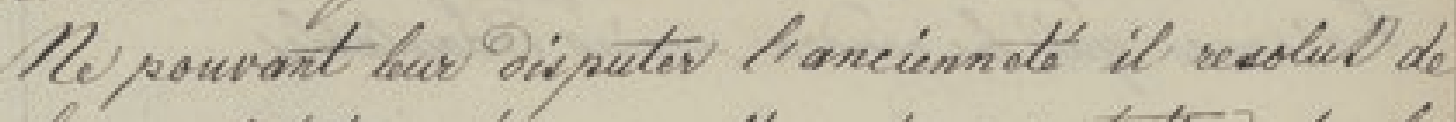
leur substituor des nonvelles de da críation of cela lai reusvit dans une grande partis gel Gurope! Les

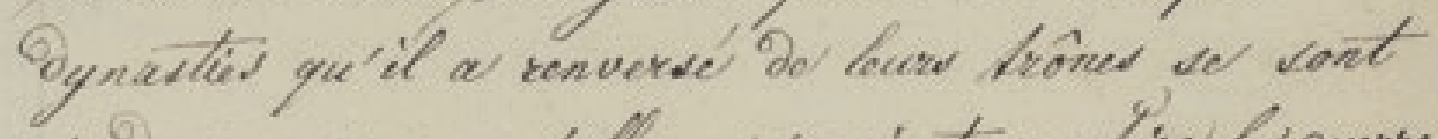
perouses, on parcequ'dles ne davaient pas gaire la quevere,

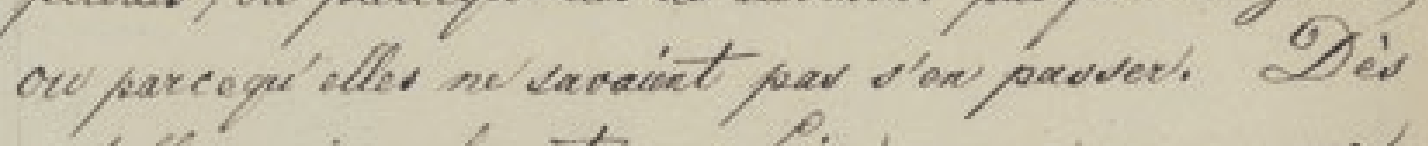
qu' dies ne voulurent pov faire cauve commene contreleur onnomi commun, ded aurent porie. Glles s'avaiont I'autre alternative que de le battre our de bie donner leurs filles en mariage - il fallait corrompre tav venite' dis qu'on ne put vesistor is sa foree - on 's'st appoese dis and trop tare de cette verité.

Quant ane fueced milituines de B.. on

a

91 
30

a ew graied tort dov'on etomner to un plus grand

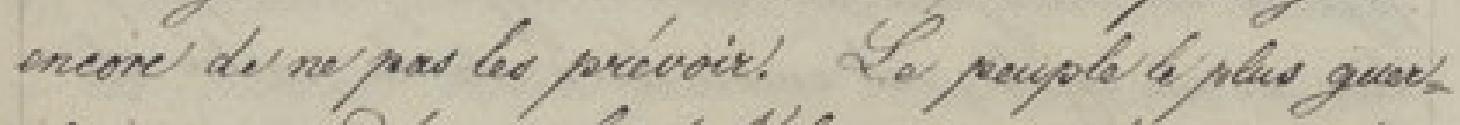
vier commandé pao lecher le sluw militacie a des

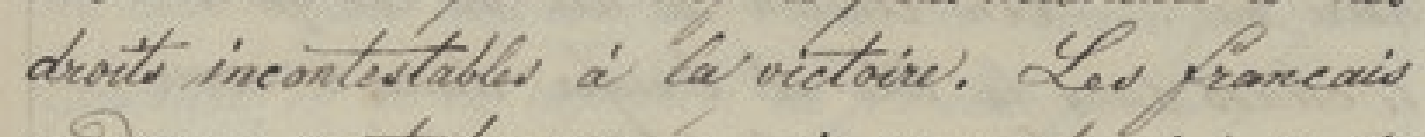
moderned cont deverus queviexs par bedoinc; c'edt lowarage de la revoliction; or un peuple pauve gowerne par un che qui dait a prececter 20 langent ne pat itre que terrible - il n'y a que le netors it liciunce qui les vondra moine sedautables.

writw 1806.

92 


\section{2}

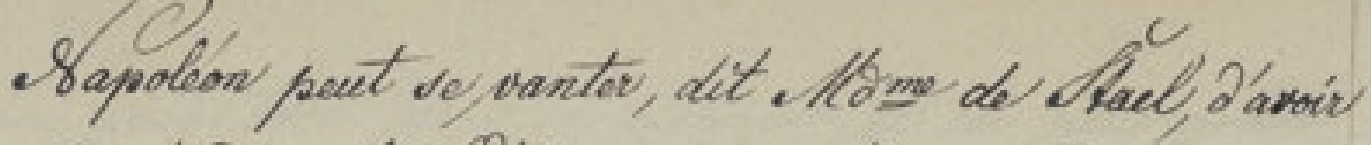
ow plue do disciples fideles a'son systome que devervitewes devous áson inporterne.

Bonaparto' "xarit per plues envic de verver le Jang qu'un homme raivonnable n'a eniré do devenuer de liargut, quand cela n'est perd necuvaise - maic da raison otwit dand da wolonte' ef fes necessites staient celles g'un sradigue.

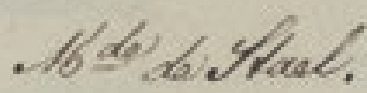




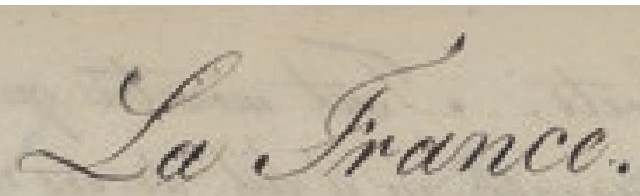

35.

19

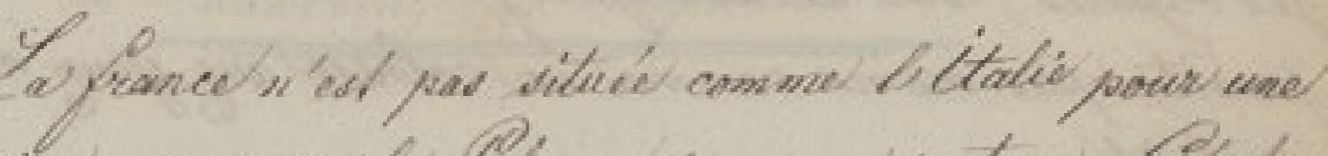
dicision - comme far Sologne pour un partage. Gost un cosposquive ufued à toule decomposition durable.

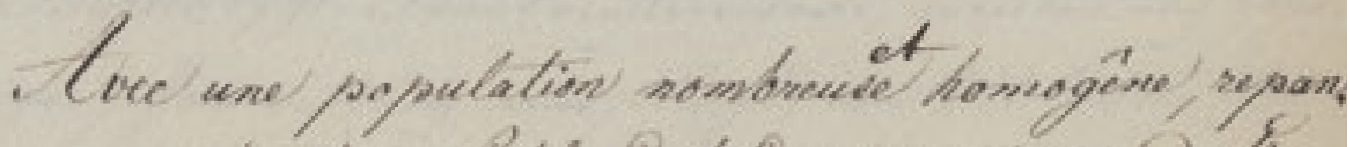

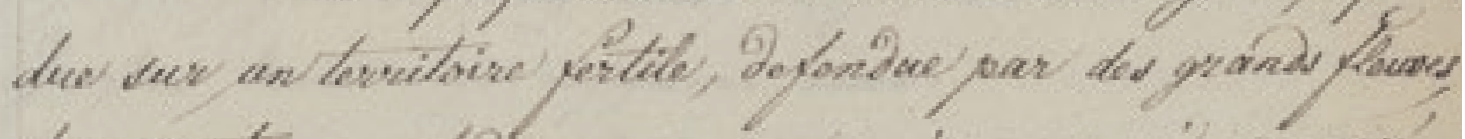

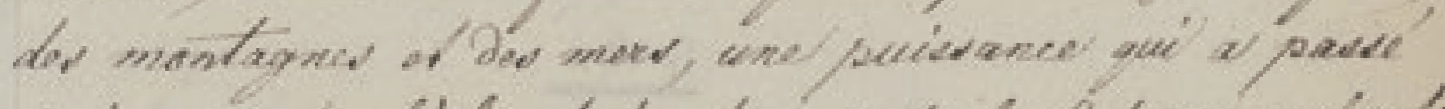

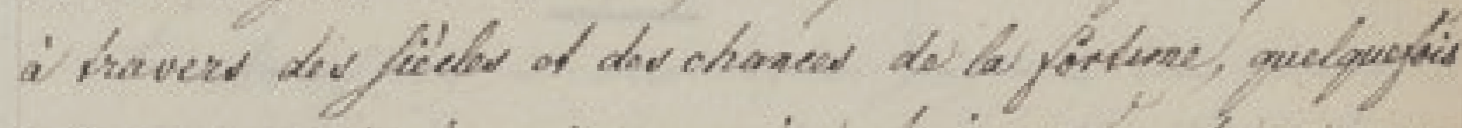

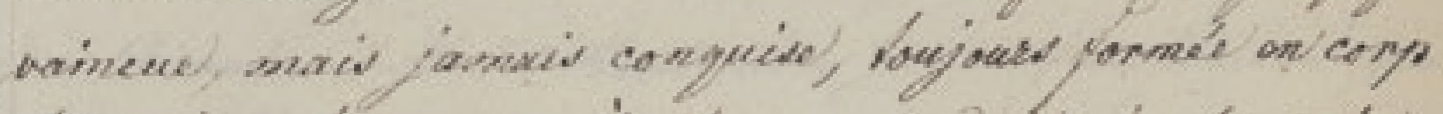

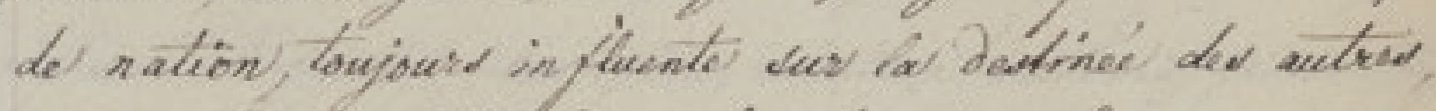

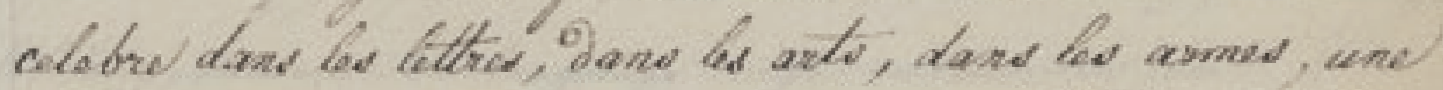
telle pusisanee eniste censme pas mecessite - avee da

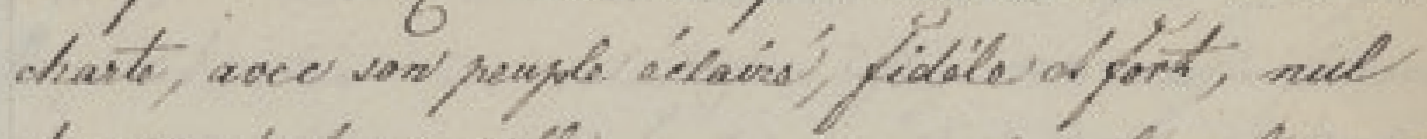

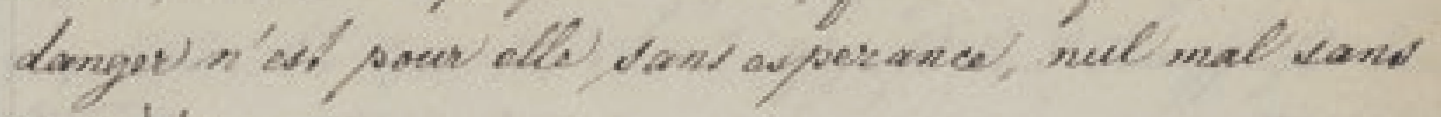
semeds.

- Ii vain, si legor, si coupable qu' on ail zupré

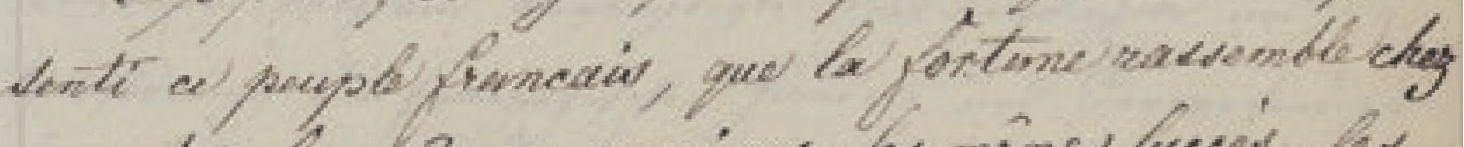
uns autre les memes prosions, les memes fueceiv, hes mesnes dosactres, lequel on. sordiaid avee/ ples dos couragel et de gloire? In moment courbe comme le rovan por las tampeto, it sestreleve comme

le

94 


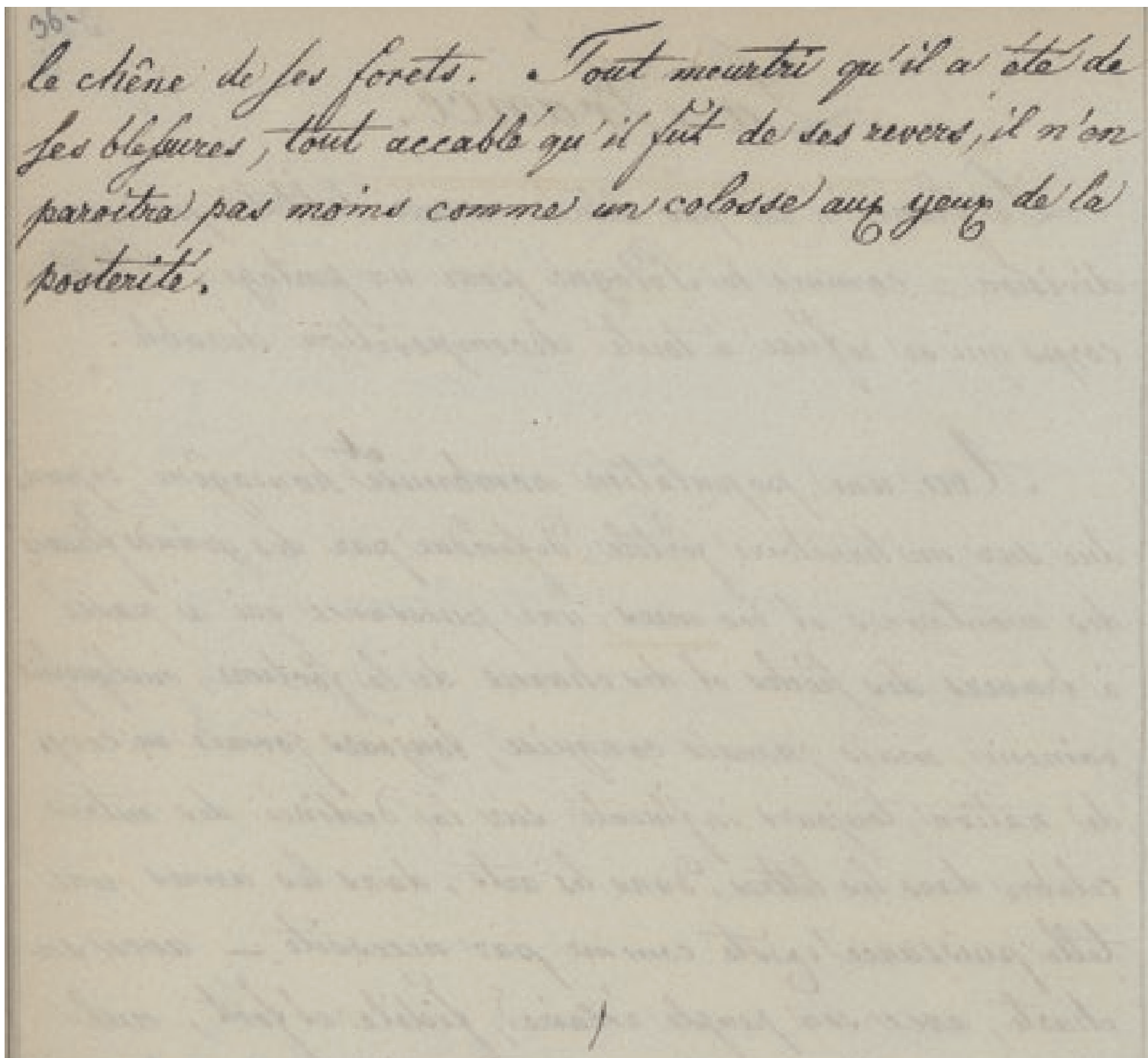

